\title{
TOPOLOGICAL APPLICATIONS OF GRADED FROBENIUS $n$-HOMOMORPHISMS, II
}

\author{
D. V. GUGNIN
}

\begin{abstract}
This paper strengthens Theorems 3.1.7 and 3.2.4 in Topological applications of graded Frobenius n-homomorphisms, by D. V, Gugnin, Tr. Mosk. Mat. Obs. 72 (2011), no. 1, 127-188; English transl., Trans. Moscow Math. Soc. 72 (2011), no. 1, 97-142. The improved version of Theorem 3.1.7 allows us to use integral techniques when working with rational cohomology algebras of $n H$-spaces. We introduce a rather broad class of even-dimensional manifolds $\mathcal{M}$ and, using integrality conditions, we show that those manifolds do not admit a 2-valued multiplication with identity. In particular, we show that complex projective spaces $\mathbb{C} P^{m}, m \geq 2$, are not $2 H$-spaces. This fact has only been known for $\mathbb{C} P^{2}$.
\end{abstract}

\section{$\S 1$. InTRODUCTION}

In Chapter 3 of [4], we investigated $n$-valued topological groups $X$ and, more generally, $n H$-spaces, $n H$-monoids, and $n H$-groups. Those spaces $X$ were subject to the following restrictions: locally contractible, paracompact, finite-dimension of rational cohomology, and a paracompact $X^{n^{2}}$. One of the goals of the present paper is to extend Theorems 3.1.7 and 3.2.4 of [4] to a less exotic, from the point of view of algebraic topology, class of spaces.

In fact, we consider Hausdorff spaces $X$ which are homotopy equivalent to a $\mathrm{CW}$ complex and such that $\operatorname{dim} H^{q}(X ; \mathbb{Q})<\infty \forall q \geq 0$. It turns out that the existence of a cellular structure (for some space homotopy equivalent to $X$ ) and, accordingly, cellular cohomology, allows for a substantial strengthening of Theorem 3.1.7.

More precisely, we show that, for an $n H$-space $X$, the diagonal $\Delta: H^{*}(X ; \mathbb{Q}) \rightarrow$ $H^{*}(X ; \mathbb{Q}) \otimes H^{*}(X ; \mathbb{Q})$ of the graded $n$-Hopf algebra sends integral rational cohomology classes $\alpha \in H^{*}(X ; \mathbb{Q})$ to integral elements of the tensor product $H^{*}(X ; \mathbb{Q}) \otimes H^{*}(X ; \mathbb{Q})$.

The obtained integrality condition allows us to show that manifolds in a rather wide class $\mathcal{M}$, introduced in this paper, do not admit a 2 -valued multiplication with identity. In particular, we show that projective spaces $\mathbb{C} P^{m}, m \geq 2$, do not admit a 2 -valued multiplication with identity. Previously, this fact had been known only for $\mathbb{C} P^{2}$ (see [2, p. 340]), even though it was announced for all $m \geq 2$. Recall, however, that $\mathbb{C} P^{m}, m \geq 1$, carries a structure of $(m+1)$ !-valued topological groups (see [1, p. 64]).

\section{$\S$ 2. Main integrality LEMma}

Consider an arbitrary compact polyhedron $X$. Let $\mathbb{K}$ be the field of coefficients, char $\mathbb{K}=0$ or $p, p>n$. In this case, one can easily compute the cohomology ring $H^{*}\left(\operatorname{Sym}^{n} X ; \mathbb{K}\right)$ of the $n$th symmetric power of $X$. The answer is given by

2010 Mathematics Subject Classification. Primary 13A02, 16T05, 55P45, 57N65.

Key words and phrases. Symmetric power, graded algebra, $n$-valued topological group, graded Frobenius $n$-homomorphism, $n$-Hopf algebra.

Supported by the RFFI grants 11-01-00694-a and 12-01-92104-YaF-a, and the RF Government Grant RF no. 2010-220-01-077, Contract 11.G34.31.0005. 
Proposition $\boldsymbol{\alpha}$. Let $X$ be a compact polyhedron, $\mathbb{K}$ a field, char $\mathbb{K}=0$ or $p, p>n$. Then the canonical projection $\pi_{n}: X^{n} \rightarrow \operatorname{Sym}^{n} X=X^{n} / S_{n}$ induces an isomorphism

$$
\pi_{n}^{*}: H^{*}\left(\operatorname{Sym}^{n} X ; \mathbb{K}\right) \rightarrow H^{*}\left(X^{n} ; \mathbb{K}\right)^{S_{n}}=\left(H^{*}(X ; \mathbb{K})^{\otimes n}\right)^{S_{n}},
$$

where $H^{*}\left(X^{n} ; \mathbb{K}\right)^{S_{n}} \subset H^{*}\left(X^{n} ; \mathbb{K}\right)$ is the subalgebra of $S_{n}$-invariant cohomology.

Proposition $\alpha$ is a consequence of the following simple result (the Transfer Theorem).

Proposition $\boldsymbol{\beta}$. Let $W$ be an arbitrary simplicial complex on which a finite group $G$ acts simplicially. Let $\mathbb{K}$ be a field, char $\mathbb{K}=0$ or $p$, and $(p,|G|)=1$. Then the projection map $\pi_{G}: W \rightarrow W / G$ induces an isomorphism $\pi_{G}^{*}: H^{*}(W / G ; \mathbb{K}) \rightarrow H^{*}(W ; \mathbb{K})^{G}$.

It follows from the general results of A. Grothendieck in Ch. 5 of [6] that Propositon $\beta$ remains true for arbitrary actions of finite groups $G$ on a paracompact $W$ if singular cohomology $H^{*}$ is replaced by Čech cohomology $\check{H}^{*}$ (for a proof, see [8, p. 319] and [7, p. 567]). For locally contractible paracompact spaces $W$, singular cohomology $H^{*}(W)$ is canonically isomorphic to Čech cohomology $\check{H}^{*}(W)$. Because of this and of the aforementioned consequence of [6], for locally contractible paracompact spaces $X$ with a paracompact $n$th degree $X^{n}$, we have an isomorphism between singular cohomology

$$
\pi_{n}^{*}: H^{*}\left(\operatorname{Sym}^{n} X ; \mathbb{K}\right) \rightarrow H^{*}\left(X^{n} ; \mathbb{K}\right)^{S_{n}},
$$

where $\mathbb{K}$ is a field, char $\mathbb{K}=0$ or $p, p>n$.

In this paper, we want to work with Hausdorff spaces $X$ which are homotopy equivalent to $\mathrm{CW}$-complexes, with an additional condition $\operatorname{dim} H^{q}(X ; \mathbb{K})<\infty \forall q \geq 0$. The proof of the existence of isomorphism $(*)$ for this class of spaces is not difficult, but the author could not find it in the literature. For this reason, it is given here. Moreover, Lemma 1 below establishes an additional integrality condition which holds for the isomorphism $\pi_{n}^{*}: H^{*}\left(\operatorname{Sym}^{n} X ; \mathbb{Q}\right) \rightarrow H^{*}\left(X^{n} ; \mathbb{Q}\right)^{S_{n}}$.

Lemma 1. Let $X$ be a Hausdorff space homotopy equivalent to a $C W$-complex and such that $\operatorname{dim} H^{q}(X ; \mathbb{K})<\infty \forall q \geq 0$, where $\mathbb{K}$ is the field of coefficients. Let $n \geq 2$ be a natural number and char $\mathbb{K}=0$ or $p, p>n$. Then:

(1) The canonical projection $\pi_{n}: X^{n} \rightarrow \operatorname{Sym}^{n} X$ induces an isomorphism

$$
\pi_{n}^{*}: H^{*}\left(\operatorname{Sym}^{n} X ; \mathbb{K}\right) \rightarrow S^{n} H^{*}(X ; \mathbb{K})=\left(H^{*}(X ; \mathbb{K})^{\otimes n}\right)^{S_{n}}=H^{*}\left(X^{n} ; \mathbb{K}\right)^{S_{n}} .
$$

(2) Let $\mathbb{K}=\mathbb{Q}$. Then for any integral rational cohomology class $\alpha \in H^{q}(X ; \mathbb{Q}), q \geq 0$, the class

$$
\alpha \otimes 1 \otimes \ldots \otimes 1+1 \otimes \alpha \otimes \ldots \otimes 1+\ldots+1 \otimes \ldots \otimes 1 \otimes \alpha \in S^{n} H^{*}(X ; \mathbb{Q})
$$

is mapped by the isomorphism $\left(\pi_{n}^{*}\right)^{-1}: S^{n} H^{*}(X ; \mathbb{Q}) \rightarrow H^{*}\left(\operatorname{Sym}^{n} X ; \mathbb{Q}\right)$ to an integral rational cohomology class in $H^{q}\left(\operatorname{Sym}^{n} X ; \mathbb{Q}\right)$.

Proof. We begin with the first part of the lemma. Since $\operatorname{Sym}^{n}$ is a homotopy functor, we may assume that $X$ is a CW-complex or even a simplicial complex, because $\mathrm{CW}$ complexes can be approximated simplicially. The space $X^{n}$ with the usual topology of the direct product decomposes into cells, which are direct products of open simplices of $X$, but in the case of an uncountable $X$, it need not be a cellular space. $X^{n}$ becomes a cellular space $\widehat{X}^{n}$ after passing to the standard completion of the topology of $X^{n}$ over all compacts.

Direct verification shows that $S_{n}$ also acts continuously on $\widehat{X}^{n}$ and that the topology of $\widehat{\operatorname{Sym}}^{n} X=\widehat{X}^{n} / S_{n}$ is obtained from that of $\operatorname{Sym}^{n} X$ by completion over compacts. The identity map $\widehat{\mathrm{Id}}_{X^{n}}: \widehat{X}^{n} \rightarrow X^{n}$ is trivially $S_{n}$-equivariant. 
For any Hausdorff space $Y$, let $\widehat{Y}$ denote the space $Y$ with the topology completed by the compacts. The identity map $\widehat{\mathrm{Id}}_{Y}: \widehat{Y} \rightarrow Y$ is a homeomorphism on compacts and therefore induces an isomorphism on (co)homology with arbitrary coefficients.

This fact and the obvious commutative diagram

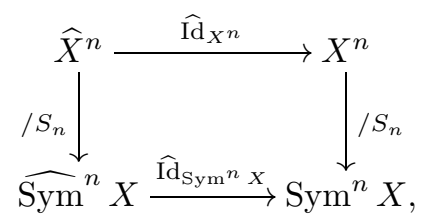

show that it suffices to prove the lemma for $\widehat{X}^{n}$ and $\widehat{\operatorname{Sym}}^{n} X$. Throughout the proof, we shall write $X^{n}$ instead of $\widehat{X}^{n}$, and $\operatorname{Sym}^{n} X$ instead of $\widehat{\operatorname{Sym}}^{n} X$, and always consider these spaces with the completed topology.

We may assume (by a theorem of Zermelo) that the set of vertices $\operatorname{Vert}(X)$ of the simplicial complex $X$ is linearly ordered. Define a partial $S_{n}$-invariant order on the vertices of $X^{n}$ by setting

$$
\begin{gathered}
\left(u^{1}, \ldots, u^{n}\right) \leq\left(v^{1}, \ldots, v^{n}\right) \Leftrightarrow u^{1} \leq v^{1}, \ldots, u^{n} \leq v^{n}, \\
\forall u^{1}, \ldots, u^{n}, v^{1}, \ldots, v^{n} \in \operatorname{Vert}(X) .
\end{gathered}
$$

As a CW-complex, $X^{n}$ admits a standard triangulation arising from the subdivision of the closed cells $\Delta_{1}^{q_{1}} \times \ldots \times \Delta_{n}^{q_{n}}$ into simplices according to the following rule. The product $\Delta_{1}^{q_{1}} \times \ldots \times \Delta_{n}^{q_{n}}$ is naturally a convex polyhedron in the affine space $\mathbb{R}^{q_{1}+\ldots+q_{n}}$. The vertices of any closed simplex $\Delta^{q} \subset \Delta_{1}^{q_{1}} \times \ldots \times \Delta_{n}^{q_{n}}$ of the desired triangulation are arbitrary linearly ordered sets of vertices

$$
\left(\left(u_{0}^{1}, \ldots, u_{0}^{n}\right)<\left(u_{1}^{1}, \ldots, u_{1}^{n}\right)<\ldots<\left(u_{q}^{1}, \ldots, u_{q}^{n}\right)\right)
$$

of $\Delta_{1}^{q_{1}} \times \ldots \times \Delta_{n}^{q_{n}}$. The closed simplex $\Delta^{q}$ is the convex hull of its vertices, and a linear order on its vertices defines a canonical orientation of it.

The original cellular structure on $X^{n}$ is invariant under the right action of $S_{n}$, $\tau\left(x_{1}, \ldots, x_{n}\right)=\left(x_{\tau(1)}, \ldots, x_{\tau(n)}\right) \forall \tau \in S_{n}, \forall x_{1}, \ldots, x_{n} \in X$. This action also preserves the partial order on the set $\operatorname{Vert}\left(X^{n}\right)$ of vertices, and the map

$$
\tau: \Delta_{1}^{q_{1}} \times \ldots \times \Delta_{n}^{q_{n}} \rightarrow \Delta_{\tau(1)}^{q_{\tau(1)}} \times \ldots \times \Delta_{\tau(n)}^{q_{\tau(n)}}, \quad \forall \tau \in S_{n},
$$

is an affine isomorphism of polyhedra.

This implies that any permutation $\tau \in S_{n}$ linearly maps an arbitrary simplex

$$
\Delta^{q}=\left[\left(u_{0}^{1}, \ldots, u_{0}^{n}\right)<\ldots<\left(u_{q}^{1}, \ldots, u_{q}^{n}\right)\right] \subset \Delta_{1}^{q_{1}} \times \ldots \times \Delta_{n}^{q_{n}}
$$

of the defined triangulation to the simplex

$$
\tau\left(\Delta^{q}\right)=\left[\left(u_{0}^{\tau(1)}, \ldots, u_{0}^{\tau(n)}\right)<\ldots<\left(u_{q}^{\tau(1)}, \ldots, u_{q}^{\tau(n)}\right)\right] \subset \Delta_{\tau(1)}^{q_{\tau(1)}} \times \ldots \times \Delta_{\tau(n)}^{q_{\tau(n)}} .
$$

Thus, the action of $S_{n}$ on $X^{n}$ is simplicial.

Let us show that, in any closed simplex $\Delta^{q}=\left[\left(u_{0}^{1}, \ldots, u_{0}^{n}\right)<\ldots<\left(u_{q}^{1}, \ldots, u_{q}^{n}\right)\right]$, there are no distinct points from the same orbit. Since the action of $S_{n}$ is simplicial, it suffices to show that no two vertices $\left(u_{i}^{1}, \ldots, u_{i}^{n}\right)$ and $\left(u_{j}^{1}, \ldots, u_{j}^{n}\right), i<j$, belong to the same orbit. This can be deduced from the following result: if $\tau\left(u^{1}, \ldots, u^{n}\right) \neq\left(u^{1}, \ldots, u^{n}\right)$, then the vertices $\tau\left(u^{1}, \ldots, u^{n}\right)$ and $\left(u^{1}, \ldots, u^{n}\right)$ are not comparable.

Indeed, suppose $\tau\left(u^{1}, \ldots, u^{n}\right)>\left(u^{1}, \ldots, u^{n}\right)$, i.e., $u^{\tau(i)} \geq u^{i}, 1 \leq i \leq n$, and for some $i_{0}, 1 \leq i_{0} \leq n, u^{\tau\left(i_{0}\right)}>u^{i_{0}}$. Then

$$
u^{\tau^{m}(i)}=u^{\tau\left(\tau^{m-1}(i)\right)} \geq u^{\tau^{m-1}(i)} \geq u^{\tau^{m-2}(i)} \geq \ldots \geq u^{\tau(i)} \quad \forall m \geq 1, \forall 1 \leq i \leq n .
$$


Therefore, $\tau^{m}\left(u^{1}, \ldots, u^{n}\right) \geq \tau\left(u^{1}, \ldots, u^{n}\right)>\left(u^{1}, \ldots, u^{n}\right) \forall m \geq 1$. This contradicts the fact that $\tau \in S_{n}$ is of finite order. Similar arguments lead to a contradiction in the case $\tau\left(u^{1}, \ldots, u^{n}\right)<\left(u^{1}, \ldots, u^{n}\right)$ as well. Thus, we have shown that the intersection of the closed simplex $\Delta^{q}=\left[\left(u_{0}^{1}, \ldots, u_{0}^{n}\right)<\ldots<\left(u_{q}^{1}, \ldots, u_{q}^{n}\right)\right]$ with the orbit $\left\{S_{n} x\right\}$ of any point $x \in X^{n}$ is either empty or consists of a single point.

This implies that the images $\pi_{n}\left(\Delta^{q}\right)$ of the simplices of $X^{n}$ under the projection $\pi_{n}: X^{n} \rightarrow \operatorname{Sym}^{n} X$ form simplices of a simplicially cellular decomposition of $\operatorname{Sym}^{n} X$, with a preimage-induced orientation.

The triangulation of $X^{n}$ can be viewed as either a simplicially cellular structure or just as a cellular structure. The canonical projection $\pi_{n}: X^{n} \rightarrow \operatorname{Sym}^{n} X$ is simplicially cellular and, moreover, the image $\pi_{n}\left(\Delta^{q}\right)$ of any simplex $\Delta^{q}$ is a simplex of $\operatorname{Sym}^{n} X$, and the restriction $\left.\pi_{n}\right|_{\Delta^{q}}: \Delta^{q} \rightarrow \pi_{n}\left(\Delta^{q}\right)$ is an orientation-preserving linear homeomorphism. Furthermore, for the open simplices, we have $\pi_{n}^{-1}\left(\pi_{n}\left(\right.\right.$ int $\left.\left.\Delta^{q}\right)\right)=\bigsqcup_{i=1}^{m} \tau_{i}\left(\right.$ int $\left.\Delta^{q}\right)$, where $S_{n}=\left\{\operatorname{Stab}_{\text {int } \Delta^{q}} \tau_{1}\right\} \sqcup \ldots \sqcup\left\{\operatorname{Stab}_{\text {int } \Delta^{q}} \tau_{m}\right\}$.

Consider cellular cochain complexes $C_{\text {simpl }}^{*}\left(X^{n}\right)$ and $C_{\text {cell }}^{*}\left(\operatorname{Sym}^{n} X\right)$ with coefficients in an arbitrary field $\mathbb{K}$ (the subscript "simpl" in $C_{\text {simpl }}^{*}\left(X^{n}\right)$ indicates that $X^{n}$ is a simplicial complex). It now follows that the cochain map $\pi_{n}^{*}: C_{\text {cell }}^{*}\left(\operatorname{Sym}^{n} X\right) \rightarrow C_{\text {simpl }}^{*}\left(X^{n}\right)$ is a monomorphism, and the image $\operatorname{Im} \pi_{n}^{*}=C_{\text {simpl }}^{*}\left(X^{n}\right)^{S_{n}}$ is the subcomplex of $S_{n}$-invariants. If we identify $C_{\text {cell }}^{*}\left(\operatorname{Sym}^{n} X\right)$ with the subcomplex $C_{\text {simpl }}^{*}\left(X^{n}\right)^{S_{n}}$ via the monomorphism $\pi_{n}^{*}$, then $\pi_{n}^{*}$ becomes the identity inclusion $i_{n}: C_{\text {simpl }}^{*}\left(X^{n}\right)^{S_{n}} \hookrightarrow C_{\text {simpl }}^{*}\left(X^{n}\right)$.

As is well known, in the case of a (co)chain complex $C^{*}$ with an action of a finite group $G$, taking homology commutes with taking the subcomplex of $G$-invariants, $H^{*}\left(C^{G}\right) \cong$ $H^{*}\left(C^{*}\right)^{G}$, whenever char $\mathbb{K}=0$ or $p,(p,|G|)=1$. This is a simple consequence of the averaging operation $\Sigma_{G}=\frac{1}{|G|} \sum_{g \in G} g$ over $G$, defined on both $C^{*}$ and $H^{*}\left(C^{*}\right)$.

Returning to the space $X^{n}$, let char $\mathbb{K}=0$ or $p, p>n$. As we said above, $i_{n}$ induces an isomorphism $i_{n}^{*}: H^{*}\left(C_{\text {simpl }}^{*}\left(X^{n}\right)^{S_{n}}\right) \cong H^{*}\left(X^{n}\right)^{S_{n}}$. This yields an isomorphism $\pi_{n}^{*}: H_{\text {cell }}^{*}\left(\operatorname{Sym}^{n} X\right) \cong H_{\text {simpl }}^{*}\left(X^{n}\right)^{S_{n}}$. Since cellular (co)homology of CW-complexes is canonically isomorphic to singular (co)homology, we have the desired isomorphism $\pi_{n}^{*}: H^{*}\left(\operatorname{Sym}^{n} X\right) \rightarrow H^{*}\left(X^{n}\right)^{S_{n}}$.

By assumption, $\operatorname{dim} H^{q}(X)<\infty \forall q \geq 0$. Therefore the Künneth formula for cohomology $H^{*}\left(X^{n}\right)=H^{*}(X)^{\otimes n}$ applies, and therefore, $H^{*}\left(X^{n}\right)^{S_{n}}=\left(H^{*}(X)^{\otimes n}\right)^{S_{n}}=$ $S^{n} H^{*}(X)$. Thus, we have an isomorphism $\pi_{n}^{*}: H^{*}\left(\operatorname{Sym}^{n} X\right) \rightarrow S^{n} H^{*}(X)$ and the first part of the lemma is proved.

Let us prove the second part. For any topological space $Y$, the integral rational cohomology classes $\alpha \in H^{*}(Y ; \mathbb{Q})$ are, by definition, the elements in the image of the homomorphism $H^{*}(Y ; \mathbb{Z}) \rightarrow H^{*}(Y ; \mathbb{Q})$ induced by the embedding $\mathbb{Z} \hookrightarrow \mathbb{Q}$. At the level of cochains, such classes are represented by the cocycles $a \in C^{*}(Y ; \mathbb{Q})$ taking on integer values on all singular simplices $\sigma: \Delta^{q} \rightarrow Y$. In the case of a CW-complex $Y$, one needs to consider the cocycles taking on integer values on all cells of $Y$.

Let $\alpha \in H_{\text {simpl }}^{q}(X ; \mathbb{Q}), q \geq 1$, be an integral rational class, $\alpha=[a]$, where $a \in$ $C_{\text {simpl }}^{q}(X ; \mathbb{Q})$ is a representing cocycle taking on integer values on all $q$-dimensional cells (simplices) $X$. We want to show that the class

$$
\begin{array}{r}
\alpha \otimes 1 \otimes \ldots \otimes 1+1 \otimes \alpha \otimes \ldots \otimes 1+\ldots+1 \otimes \ldots \otimes 1 \otimes \alpha \in S^{n} H^{*}(X ; \mathbb{Q}) \cong \\
H^{*}\left(\operatorname{Sym}^{n} X ; \mathbb{Q}\right)
\end{array}
$$

is integral in $H^{*}\left(\operatorname{Sym}^{n} X ; \mathbb{Q}\right)$ (the case $q=0$ being trivial).

Let $C_{\text {cell }}^{*}\left(X^{n} ; \mathbb{Q}\right)$ denote the cochain complex on the CW-complex $X^{n}$ whose cells are products of open simplices of $X$. As before, let $C_{\text {simpl }}^{*}\left(X^{n} ; \mathbb{Q}\right)$ denote the cochain complex of the simplicial complex $X^{n}$ with the above triangulation. For cell cochains, one has 
the $\times$-product. Let $a_{1} \in C_{\text {simpl }}^{q_{1}}(X ; \mathbb{Q}), \ldots, a_{n} \in C_{\text {simpl }}^{q_{n}}(X ; \mathbb{Q})$. Then

$$
\begin{aligned}
a_{1} \times \ldots \times a_{n}\left(\Delta_{1}^{p_{1}} \times \ldots \times \Delta_{n}^{p_{n}}\right)=(-1)^{q_{2} p_{1}+q_{3}\left(p_{1}+p_{2}\right)+\ldots+q_{n}\left(p_{1}+p_{2}+\ldots+p_{n-1}\right)} \times \\
\\
a_{1}\left(\Delta_{1}^{p_{1}}\right) a_{2}\left(\Delta_{2}^{p_{2}}\right) \ldots a_{n}\left(\Delta_{n}^{p_{n}}\right), \quad a_{1} \times \ldots \times a_{n} \in C_{\text {cell }}^{q_{1}+\ldots+q_{n}}\left(X^{n} ; \mathbb{Q}\right) .
\end{aligned}
$$

The element $\alpha_{(n)}:=\alpha \otimes 1 \otimes \ldots \otimes 1+\ldots+1 \otimes \ldots \otimes 1 \otimes \alpha \in H_{\text {cell }}^{q}\left(X^{n} ; \mathbb{Q}\right)$ is represented by the cocycle $a_{(n)}:=a \times 1 \times \ldots \times 1+\ldots+1 \times \ldots \times 1 \times a \in C_{\text {cell }}^{q}\left(X^{n} ; \mathbb{Q}\right)$. Now we shall produce an $S_{n}$-invariant integral rational cocycle $\widetilde{a}_{(n)} \in C_{\text {simpl }}^{q}\left(X^{n} ; \mathbb{Q}\right)^{S_{n}}$ such that its cohomology class $\left[\widetilde{a}_{(n)}\right]=\widetilde{\alpha}_{(n)} \in H_{\text {simpl }}^{q}\left(X^{n} ; \mathbb{Q}\right)$ corresponds to $\alpha_{(n)} \in H_{\text {cell }}^{q}\left(X^{n} ; \mathbb{Q}\right)$ under the canonical isomorphism $\psi_{\text {simpl-cell }}: H_{\text {simpl }}^{*}\left(X^{n} ; \mathbb{Q}\right) \rightarrow H_{\text {cell }}^{*}\left(X^{n} ; \mathbb{Q}\right)$.

The smaller simplicial structure on $X^{n}$ is a refinement of the cell structure. As is known, this implies that $\psi_{\text {simpl-cell }}\left(\widetilde{\alpha}_{(n)}\right)=\alpha_{(n)}$ if, for any $q$-dimensional cell $\Delta_{1}^{q_{1}} \times \ldots \times$ $\Delta_{n}^{q_{n}}, q_{1}+\ldots+q_{n}=q$, of the cellular structure on $X^{n}$, the value $a_{(n)}\left(\Delta_{1}^{q_{1}} \times \ldots \times \Delta_{n}^{q_{n}}\right)$ coincides with the sum of the values of the cocycle $\widetilde{a}_{(n)}$ on the $q$-dimensional simplices comprising the cell $\Delta_{1}^{q_{1}} \times \ldots \times \Delta_{n}^{q_{n}}$.

Define a cochain $\widetilde{a}_{(n)} \in C_{\text {simpl }}^{q}\left(X^{n} ; \mathbb{Q}\right)$ by the formula

$$
\begin{aligned}
& \widetilde{a}_{(n)}\left[\left(u_{0}^{1}, \ldots, u_{0}^{n}\right)<\left(u_{1}^{1}, \ldots, u_{1}^{n}\right)<\ldots<\left(u_{q}^{1}, \ldots, u_{q}^{n}\right)\right]= \\
& a\left(\Delta\left[u_{0}^{1}, u_{1}^{1}, \ldots, u_{q}^{1}\right]\right)+a\left(\Delta\left[u_{0}^{2}, u_{1}^{2}, \ldots, u_{q}^{2}\right]\right)+\ldots+a\left(\Delta\left[u_{0}^{n}, u_{1}^{n}, \ldots, u_{q}^{n}\right]\right),
\end{aligned}
$$

where we set $a\left(\Delta\left[u_{0}^{j}, u_{1}^{j}, \ldots, u_{q}^{j}\right]\right)=0$ if there are repetitions among the vertices $u_{0}^{j} \leq$ $u_{1}^{j} \leq \ldots \leq u_{q}^{j}, 1 \leq j \leq n$. In the case $u_{0}^{j}<u_{1}^{j}<\ldots<u_{q}^{j}$, the expression $\Delta\left[u_{0}^{j}, u_{1}^{j}, \ldots, u_{q}^{j}\right]$ denotes the $q$-dimensional simplex of $X$ on the vertices $u_{0}^{j}, \ldots, u_{q}^{j}, 1 \leq j \leq n$, with the (canonical) orientation determined by the ordering $u_{0}^{j}<\ldots<u_{q}^{j}$.

Clearly, the cochain $\widetilde{a}_{(n)}$ is integral and $S_{n}$-invariant. Let us check the cocycle condition $\delta \widetilde{a}_{(n)}=0$. We have $\delta \widetilde{a}_{(n)}\left(\Delta^{q+1}\right)= \pm \widetilde{a}_{(n)}\left(\partial \Delta^{q+1}\right)$ for any $(q+1)$-dimensional simplex $\Delta^{(q+1)}=\left[\left(u_{0}^{1}, \ldots, u_{0}^{n}\right)<\ldots<\left(u_{q+1}^{1}, \ldots, u_{q+1}^{n}\right)\right]$ of the simplicial complex $X^{n}$.

The boundary of $\Delta^{(q+1)}$ is of the form

$$
\partial \Delta^{q+1}=\sum_{s=0}^{q+1}(-1)^{s}\left[\left(u_{0}^{1}, \ldots, u_{0}^{n}\right)<\ldots<\left(\widehat{u_{s}^{1}, \ldots, u_{s}^{n}}\right)<\ldots<\left(u_{q+1}^{1}, \ldots, u_{q+1}^{n}\right)\right] .
$$

We then have

$$
\begin{aligned}
& \widetilde{a}_{(n)}\left(\partial \Delta^{q+1}\right)= \\
& \sum_{s=0}^{q+1}(-1)^{s}\left(a\left(\Delta\left[u_{0}^{1}, \ldots, \widehat{u_{s}^{1}}, \ldots, u_{q+1}^{1}\right]\right)+\ldots+a\left(\Delta\left[u_{0}^{n}, \ldots, \widehat{u_{s}^{n}}, \ldots, u_{q+1}^{n}\right]\right)\right)= \\
& \quad \sum_{k=1}^{n} a\left(\sum_{s=0}^{q+1}(-1)^{s} \Delta\left[u_{0}^{k}, \ldots, \widehat{u_{s}^{k}}, \ldots, u_{q+1}^{k}\right]\right)=\sum_{k=1}^{n} a\left(\partial \Delta\left[u_{0}^{k}, \ldots, u_{q+1}^{k}\right]\right)=0 .
\end{aligned}
$$

Therefore, $\widetilde{a}_{(n)} \in C_{\text {simpl }}^{q}\left(X^{n} ; \mathbb{Q}\right)$ is a cocycle. Now we want to show that the cohomology class $\left[\widetilde{a}_{(n)}\right]=\widetilde{\alpha}_{(n)} \in H_{\text {simpl }}^{q}\left(X^{n} ; \mathbb{Q}\right)$ is mapped to the class $\alpha_{(n)} \in H_{\text {cell }}^{q}\left(X^{n} ; \mathbb{Q}\right)$ under the canonical isomorphism $H_{\text {simpl }}^{q}\left(X^{n} ; \mathbb{Q}\right) \cong H_{\text {cell }}^{q}\left(X^{n} ; \mathbb{Q}\right)$. As before, it suffices to show that for any cell $\Delta_{1}^{q_{1}} \times \ldots \times \Delta_{n}^{q_{n}}, q_{1}+\ldots+q_{n}=q$, of $X^{n}$ the value $a_{(n)}\left(\Delta_{1}^{q_{1}} \times \ldots \times \Delta_{n}^{q_{n}}\right)$ coincides with the sum of the values of the cocycle $\widetilde{a}_{(n)}$ on the $q$-dimensional simplices comprising the cell $\Delta_{1}^{q_{1}} \times \ldots \times \Delta_{n}^{q_{n}}$. We shall refer to this condition as the coherence condition.

Fix an arbitrary $q$-dimensional cell $\Delta_{1}^{q_{1}} \times \ldots \times \Delta_{n}^{q_{n}}, q_{1}+\ldots+q_{n}=q$, of $X^{n}$. We have two cases: (A) $q_{k}<q \forall 1 \leq k \leq n$, and (B) $\exists$ ! $i, 1 \leq i \leq n, q_{i}=q, q_{j}=0 \forall j \neq i$. 
Case (A). Since $a_{(n)}=a \times 1 \times \ldots \times 1+\ldots+1 \times \ldots \times 1 \times a$, we have $a_{(n)}\left(\Delta_{1}^{q_{1}} \times \ldots \times\right.$ $\left.\Delta_{n}^{q_{n}}\right)=0$. Consider an arbitrary simplex $\Delta^{q}\left[\left(u_{0}^{1}, \ldots, u_{0}^{n}\right)<\ldots<\left(u_{q}^{1}, \ldots, u_{q}^{n}\right)\right]$ in the cell $\Delta_{1}^{q_{1}} \times \ldots \times \Delta_{n}^{q_{n}}$. Since $q_{k}<q \forall 1 \leq k \leq n$, among the vertices $u_{0}^{k} \leq u_{1}^{k} \leq \ldots \leq u_{q}^{k}$ of $\Delta_{k}^{q_{k}}$ there are always repetitions for any $k, 1 \leq k \leq n$. Therefore,

$$
\widetilde{a}_{(n)}\left(\Delta^{q}\left[\left(u_{0}^{1}, \ldots, u_{0}^{n}\right)<\ldots<\left(u_{q}^{1}, \ldots, u_{q}^{n}\right)\right]\right)=\sum_{k=1}^{n} a\left(\Delta\left[u_{0}^{k}, \ldots, u_{q}^{k}\right]\right)=\sum_{k=1}^{n} 0=0,
$$

and, in this case, the coherence condition is trivially true.

Case (B). Let $q_{i}=q$ for some $i, 1 \leq i \leq n$. Then $q_{j}=0$ for any $j \neq i, 1 \leq j \leq n$. Therefore, the cell

$$
\Delta_{1}^{q_{1}} \times \ldots \times \Delta_{n}^{q_{n}}=\mathrm{pt}_{1} \times \ldots \times \Delta_{i}^{q}\left[u_{0}^{i}, u_{1}^{i}, \ldots, u_{q}^{i}\right] \times \ldots \times \mathrm{pt}_{n}
$$

is a $q$-dimensional simplex parallel to the $i$-factor of $X^{n}$. It is easy to see that

$$
a_{(n)}\left(\mathrm{pt}_{1} \times \ldots \times \Delta_{i}^{q}\left[u_{0}^{i}, u_{1}^{i}, \ldots, u_{q}^{i}\right] \times \ldots \times \mathrm{pt}_{n}\right)=a\left(\Delta_{i}^{q}\left[u_{0}^{i}, u_{1}^{i}, \ldots, u_{q}^{i}\right]\right) .
$$

Since the simplex $\mathrm{pt}_{1} \times \ldots \times \Delta_{i}^{q} \times \ldots \times \mathrm{pt}_{n}$ does not break up when passing to the simplicial complex $X^{n}$, it remains to compute

$$
\widetilde{a}_{(n)}\left(\mathrm{pt}_{1} \times \ldots \times \Delta_{i}^{q} \times \ldots \times \mathrm{pt}_{n}\right)=\sum_{k=1}^{n} a\left(\Delta\left[u_{0}^{k}, u_{1}^{k}, \ldots, u_{q}^{k}\right]\right)=a\left(\Delta_{i}^{q}\left[u_{0}^{i}, u_{1}^{i}, \ldots, u_{q}^{i}\right]\right) .
$$

Therefore, the coherence condition

$$
a_{(n)}\left(\mathrm{pt}_{1} \times \ldots \times \Delta_{i}^{q} \times \ldots \times \mathrm{pt}_{n}\right)=\widetilde{a}_{(n)}\left(\mathrm{pt}_{1} \times \ldots \times \Delta_{i}^{q} \times \ldots \times \mathrm{pt}_{n}\right)
$$

is true in this case as well. Thus, the coherence condition for the cocycles $\widetilde{a}_{(n)}$ and $a_{(n)}$ is always true.

In summary, we have the following. We have constructed an $S_{n}$-invariant integral rational cocycle $\widetilde{a}_{(n)} \in C_{\text {simpl }}^{q}\left(X^{n} ; \mathbb{Q}\right)^{S_{n}}$ whose cohomology class $\left[\widetilde{a}_{(n)}\right] \in H_{\text {simpl }}^{q}\left(X^{n} ; \mathbb{Q}\right)^{S_{n}}$ corresponds to the class $\alpha \otimes 1 \otimes \ldots \otimes 1+\ldots+1 \otimes \ldots \otimes 1 \otimes \alpha \in S^{n} H^{*}(X ; \mathbb{Q})$. But because we have, at the level of cochain complexes, the canonical isomorphism $C_{\text {simpl }}^{q}\left(X^{n} ; \mathbb{Q}\right)^{S_{n}} \cong$ $C_{\text {cell }}^{q}\left(\operatorname{Sym}^{n} X ; \mathbb{Q}\right)$ (sending integral cochains to integral cochains), we may assume that the integral rational cocycle $\widetilde{a}_{(n)}$ lies in $C_{\text {cell }}^{q}\left(\operatorname{Sym}^{n} X ; \mathbb{Q}\right)$. Moreover, its cohomology class $\left[\widetilde{a}_{(n)}\right] \in H_{\text {cell }}^{q}\left(\operatorname{Sym}^{n} X ; \mathbb{Q}\right)$ is integral and is mapped to the class $\alpha \otimes 1 \otimes \ldots \otimes 1+\ldots+$ $1 \otimes \ldots \otimes 1 \otimes \alpha \in S^{n} H^{*}(X ; \mathbb{Q})$ under the isomorphism $H^{*}\left(\operatorname{Sym}^{n} X ; \mathbb{Q}\right) \cong S^{n} H^{*}(X ; \mathbb{Q})$ constructed above. This finishes the proof of the lemma.

\section{§ 3. A strengthening of Theorems 3.1.7 And 3.2.4}

We want to prove the following strengthening of Theorem 3.1.7 of [4].

Theorem 1. (1) Let $X$ be a connected Hausdorff space homotopy equivalent to a $C W$ complex, and such that $\operatorname{dim} H^{q}(X ; \mathbb{Q})<\infty \forall q \geq 0$. If $X$ has a structure of $n H$-space, then the algebra $H^{*}(X ; \mathbb{Q})$ has a structure of graded $n$-Hopf prealgebras.

(2) Let $X$ be a connected Hausdorff space homotopy equivalent to a countable $C W$ complex, and such that $\operatorname{dim} H^{q}(X ; \mathbb{Q})<\infty \forall q \geq 0$. If $X$ has a structure of a $n H$-monoid $(n H$-group $)$, then the algebra $H^{*}(X ; \mathbb{Q})$ has a structure of a graded n-bialgebra $(n$-Hopf algebra). 
In both cases, if the groups $H_{q}(X ; \mathbb{Z})$ are finitely generated for all $q \geq 0$, then the diagonal $\Delta: H^{*}(X ; \mathbb{Q}) \rightarrow H^{*}(X ; \mathbb{Q}) \otimes H^{*}(X ; \mathbb{Q})$ of the graded $n$-Hopf prealgebra $(n$ bialgebra, $n$-Hopf algebra) $H^{*}(X ; \mathbb{Q})$ maps integral rational classes $\alpha \in H^{*}(X ; \mathbb{Q})$ to integral elements of $H^{*}(X ; \mathbb{Q}) \otimes H^{*}(X ; \mathbb{Q})$.

Proof. (1) The proof of Theorem 3.1.7 (which is an obvious modification of that of Theorem 3.1.5) is completely applicable in this case because of the isomorphism

$$
\pi_{n}^{*}: H^{*}\left(\operatorname{Sym}^{n} X ; \mathbb{Q}\right) \rightarrow S^{n} H^{*}(X ; \mathbb{Q})=H^{*}\left(X^{n} ; \mathbb{Q}\right)^{S_{n}}
$$

where $\pi_{n}: X^{n} \rightarrow \operatorname{Sym}^{n} X$ is the canonical projection. The isomorphism $\pi_{n}^{*}$ holds because of Lemma 1.

(2) The only thing needed to use the proof of Theorem 3.1.7 again is an isomorphism $\pi_{n(Y)}^{*}: H^{*}\left(\operatorname{Sym}^{n} Y ; \mathbb{Q}\right) \rightarrow S^{n} H^{*}(Y ; \mathbb{Q})$ for $Y=X \times X$ and $Y=\operatorname{Sym}^{n} X$. By Lemma 1 , it suffices that $Y$ be a Hausdorff space homotopy equivalent to a $\mathrm{CW}$-complex and have finite-dimensional rational cohomology in each dimension: $\operatorname{dim} H^{q}(Y ; \mathbb{Q})<\infty \forall q \geq 0$. The first and the third conditions are obviously satisfied for both $X \times X$ and $\operatorname{Sym}^{n} X$.

Let us check the homotopy equivalence. By assumption, $X$ is homotopy equivalent to a countable CW-complex $Z$. This gives rise to a homotopy equivalence $X \times X \sim Z \times Z$, where $Z \times Z$ is a $C W$-complex as a finite product of countable $C W$-complexes.

It remains to consider the case $Y=\operatorname{Sym}^{n} X$. Since a countable $\mathrm{CW}$-complex has a countable simplicial approximation, we may assume that $Z$ is a countable simplicial complex. Because of that, the space $Z^{n}$ is a $C W$-complex with products of open simplices of $Z$ as cells.

Linearly ordering the set $\operatorname{Vert}(Z)$ of vertices of $Z$ and applying the construction from the proof of Lemma 1, we can refine the original cellular structure on $Z^{n}$ to obtain a simplicial decomposition invariant under the action of $S_{n}$. The "weak topology" axiom, which holds for the cellular structure on $Z^{n}$, automatically holds for the simplicial decomposition. Thus, $Z^{n}$ has an $S_{n}$-invariant triangulation.

It is known that, for simplicial actions of an arbitrary finite group $G$ on an arbitrary simplicial complex $W$, the quotient $W / G$ is a simplicial complex. The simplices of $W / G$ are the images of the simplices of the second barycentric subdivision of $W$ under the projection $\pi_{G}: W \rightarrow W / G$. As we observed above, the space $\operatorname{Sym}^{n} Z=Z^{n} / S_{n}$ can be triangulated. Since $\mathrm{Sym}^{n}$ is a homotopy functor, the space $\operatorname{Sym}^{n} X$ is homotopy equivalent to the simplicial complex $\operatorname{Sym}^{n} Z$.

Thus, in both cases $Y=X \times X$ and $Y=\operatorname{Sym}^{n} X$, we have checked all three conditions guaranteeing an isomorphism $\pi_{n(Y)}^{*}: H^{*}\left(\operatorname{Sym}^{n} Y ; \mathbb{Q}\right) \rightarrow S^{n} H^{*}(Y ; \mathbb{Q})$. The isomorphisms $\pi_{n(X \times X)}^{*}$ and $\pi_{n\left(\operatorname{Sym}^{n} X\right)}^{*}$, needed for the proof of the coassociativity of the diagonal $\Delta: H^{*}(X ; \mathbb{Q}) \rightarrow H^{*}(X ; \mathbb{Q}) \otimes H^{*}(X ; \mathbb{Q})$, allow us to reduce the proof of the second part of Theorem 1 to a verbatim repetition of the proof of Theorem 3.1.7.

In both parts of Theorem 1 , the diagonal $\Delta: H^{*}(X ; \mathbb{Q}) \rightarrow H^{*}(X ; \mathbb{Q}) \otimes H^{*}(X ; \mathbb{Q})$ is constructed as $\Delta=\mu^{*} \circ\left(\pi_{n}^{*}\right)^{-1} \circ \chi_{n}$, where $\chi_{n}: H^{*}(X ; \mathbb{Q}) \rightarrow S^{n} H^{*}(X ; \mathbb{Q}), \chi_{n}(\alpha)=$ $\alpha \otimes 1 \otimes \ldots \otimes 1+\ldots+1 \otimes \ldots \otimes 1 \otimes \alpha$ is a natural $n$-homomorphism of the algebra $H^{*}(X ; \mathbb{Q}), \pi_{n}^{*}: H^{*}\left(\operatorname{Sym}^{n} X ; \mathbb{Q}\right) \rightarrow S^{n} H^{*}(X ; \mathbb{Q})$ is the canonical isomorphism, and $\mu^{*}: H^{*}\left(\operatorname{Sym}^{n} X ; \mathbb{Q}\right) \rightarrow H^{*}\left(X^{2} ; \mathbb{Q}\right)=H^{*}(X ; \mathbb{Q}) \otimes H^{*}(X ; \mathbb{Q})$ is the algebra homomorphism induced by the $n$-valued multiplication $\mu: X^{2} \rightarrow \operatorname{Sym}^{n} X$.

By Lemma 1 , if $\alpha \in H^{q}(X ; \mathbb{Q}), q \geq 0$, is an integral element, then $\left(\pi_{n}^{*}\right)^{-1} \circ \chi_{n}(\alpha) \in$ $H^{*}\left(\operatorname{Sym}^{n} X ; \mathbb{Q}\right)$ is also integral. Since the homomorphism $\mu^{*}: H^{*}\left(\operatorname{Sym}^{n} X ; \mathbb{Q}\right) \rightarrow$ $H^{*}\left(X^{2} ; \mathbb{Q}\right)$ maps integral elements to integral elements, $\mu^{*} \circ\left(\pi_{n}^{*}\right)^{-1} \circ \chi_{n}(\alpha)=\Delta(\alpha) \in$ $H^{*}\left(X^{2} ; \mathbb{Q}\right)$ is integral. 
Suppose now that the groups $H_{q}(X ; \mathbb{Z})$ are finitely generated for all $q \geq 0$. Then the cohomology Künneth formula

$$
\begin{aligned}
H^{q}(X \times X ; \mathbb{Z})=( & \left.\bigoplus_{i+j=q} H^{i}(X ; \mathbb{Z}) \otimes H^{j}(X ; \mathbb{Z})\right) \\
& 1 \oplus\left(\bigoplus_{i+j=q+1} \operatorname{Tor}\left(H^{i}(X ; \mathbb{Z}), H^{j}(X ; \mathbb{Z})\right)\right),
\end{aligned}
$$

applies, where the first summand is natural and is the image of the $\times$-product

$$
\nu_{\mathbb{Z}}: H^{*}(X ; \mathbb{Z}) \otimes H^{*}(X ; \mathbb{Z}) \rightarrow H^{*}(X \times X ; \mathbb{Z}) .
$$

Since the integral $\times$-product $\nu_{\mathbb{Z}}$ becomes the rational $\times$-product

$$
\nu_{\mathbb{Q}}: H^{*}(X ; \mathbb{Q}) \otimes H^{*}(X ; \mathbb{Q}) \rightarrow H^{*}(X \times X ; \mathbb{Q})
$$

under the natural homomorphism of the coefficient rings $\mathbb{Z} \hookrightarrow \mathbb{Q}$, the integral elements of $H^{*}(X \times X ; \mathbb{Q})$ are exactly the integral elements of the tensor square $H^{*}(X ; \mathbb{Q}) \otimes$ $H^{*}(X ; \mathbb{Q})$. Thus, the element $\Delta(\alpha) \in H^{*}(X ; \mathbb{Q}) \otimes H^{*}(X ; \mathbb{Q})$ is integral for any integral $\alpha \in H^{*}(X ; \mathbb{Q})$. The theorem is proved.

Now we want to strengthen Theorem 3.2.4.

Theorem 2. Let $X$ be a connected Hausdorff space homotopy equivalent to a $C W$ complex and such that $\operatorname{dim} H^{q}(X ; \mathbb{Q})<\infty \forall q \geq 0$. If $X$ admits a structure of a $2 H$-space, then its fundamental group $\pi_{1}(X)$ does not belong to the class $\mathcal{C}$.

Proof. Assume the opposite. Let $X$ be a $2 H$-space and $G:=\pi_{1}(X) \in \mathcal{C}$. By Theorem 1 , $H^{*}(X ; \mathbb{Q})$ is a graded 2-Hopf prealgebra. This means that there is a 2-homomorphism $\Delta: H^{*}(X ; \mathbb{Q}) \rightarrow H^{*}(X ; \mathbb{Q}) \otimes H^{*}(X ; \mathbb{Q})$ satisfying the counit condition: $\Delta(u)=2 u \otimes 1+$ $1 \otimes 2 u+\sum_{i=1}^{N} u_{i}^{\prime} \otimes u_{i}^{\prime \prime},\left|u_{i}^{\prime}\right| \geq 1,\left|u_{i}^{\prime \prime}\right| \geq 1,1 \leq i \leq N$ for any $u \in H^{*}(X ; \mathbb{Q}),|u| \geq 1$. Suppose a CW-complex $Y$ is homotopy equivalent to $X$ and $f: X \rightarrow Y$ is a homotopy equivalence.

Consider a cellular realization of $B G=K(G, 1)$. Since $G \in \mathcal{C}$, there are a basis $\alpha_{1}, \ldots, \alpha_{2 g}, \beta_{1}, \ldots, \beta_{s} \in H^{1}(B G ; \mathbb{Q}), g \geq 2, s \geq 0$, and a nonzero element $\gamma \in H^{2}(B G ; \mathbb{Q})$ such that both conditions of Definition 3.2.1 of 4 of the class $\mathcal{C}$ are satisfied. Fix base points $y_{0} \in Y$ and $b_{0} \in B G$. It is well known from homotopy theory of cellular spaces that there is a unique (up to homotopy of pointed spaces) continuous map $h_{\varphi}:\left(Y, y_{0}\right) \rightarrow$ $\left(B G, b_{0}\right)$ inducing an arbitrary isomorphism $\varphi: \pi_{1}(Y)=\pi_{1}(X) \rightarrow G=\pi_{1}(B G)$.

Fix an isomorphism $\varphi_{0}: \pi_{1}(Y) \rightarrow G$ and let $h=h_{\varphi_{0}}:\left(Y, y_{0}\right) \rightarrow\left(B G, b_{0}\right)$. Since $h_{*}=\varphi_{0}: \pi_{1}(Y) \rightarrow \pi_{1}(B G)$ is an isomorphism, $h_{*}: H_{1}(Y ; \mathbb{Z}) \rightarrow H_{1}(B G ; \mathbb{Z})$ is also an isomorphism. Therefore, we also have isomorphisms $h_{*}: H_{1}(Y ; \mathbb{Q}) \rightarrow H_{1}(B G ; \mathbb{Q})$ and $h^{*}: H^{1}(B G ; \mathbb{Q}) \rightarrow H^{1}(Y ; \mathbb{Q})$.

By a theorem of Hopf, the map $h_{*}: H_{2}(Y ; \mathbb{Z}) \rightarrow H_{2}(B G ; \mathbb{Z})$ is an epimorphism whose kernel coincides with the image of the Hurewicz homomorphism $\pi_{2}(Y) \rightarrow H_{2}(Y ; \mathbb{Z})$. This implies that $h_{*}: H_{2}(Y ; \mathbb{Q}) \rightarrow H_{2}(B G ; \mathbb{Q})$ is an epimorphism, and hence $h^{*}: H^{2}(B G ; \mathbb{Q}) \rightarrow$ $H^{2}(Y ; \mathbb{Q})$ is a monomorphism.

Since $h^{*}: H^{*}(B G ; \mathbb{Q}) \rightarrow H^{*}(Y ; \mathbb{Q})$ is an algebra homomorphism inducing an isomorphism on $H^{1}$ and a monomorphism on $H^{2}$, both conditions of Definition 3.2.1 are satisfied for the basis $h^{*}\left(\alpha_{1}\right), \ldots, h^{*}\left(\alpha_{2 g}\right), h^{*}\left(\beta_{1}\right), \ldots, h^{*}\left(\beta_{s}\right) \in H^{1}(Y ; \mathbb{Q})$ and the nonzero element $h^{*}(\gamma) \in H^{2}(Y ; \mathbb{Q})$. Since the homotopy equivalence $f: X \rightarrow Y$ induces an algebra isomorphism $f^{*}: H^{*}(Y ; \mathbb{Q}) \rightarrow H^{*}(X ; \mathbb{Q})$, both conditions of Definition 3.2.1 are also satisfied for the basis $\bar{\alpha}_{1}=f^{*} \circ h^{*}\left(\alpha_{1}\right), \ldots, \bar{\alpha}_{2 g}=f^{*} \circ h^{*}\left(\alpha_{2 g}\right), \bar{\beta}_{1}=f^{*} \circ h^{*}\left(\beta_{1}\right), \ldots$, $\bar{\beta}_{s}=f^{*} \circ h^{*}\left(\beta_{s}\right) \in H^{1}(X ; \mathbb{Q})$ and the nonzero element $\bar{\gamma}=f^{*} \circ h^{*}(\gamma) \in H^{2}(X ; \mathbb{Q})$. Denote the algebra $H^{*}(X ; \mathbb{Q})$ by $A^{*}$. 
In summary, we have an algebra $A^{*}$, a basis $\bar{\alpha}_{1}, \ldots, \bar{\alpha}_{2 g}, \bar{\beta}_{1}, \ldots, \bar{\beta}_{s} \in A^{1}, g \geq 2, s \geq 0$, and a nonzero element $\bar{\gamma} \in A^{2}$ satisfying the conditions of Definition 3.2.1, and we also have a 2-homomorphism $\Delta: A^{*} \rightarrow A^{*} \otimes A^{*}$ satisfying the counit axiom. Now, using the same arguments as in the proof of Theorem 3.2.4, we have $g=-3$, a contradiction. The theorem is proved.

\section{$\S$ 4. A Class $\mathcal{M}$ of eVen-Dimensional manifolds Which are not $2 H$-SPaCes}

Definition 1. We say that a connected closed orientable topological $2 n$-dimensional manifold $M^{2 n}, n \neq 1,2,4$, belongs to the class $\mathcal{M}$, if the first betti number of $M^{2 n}$ is zero, $b_{1}\left(M^{2 n}\right)=0$, and there is an element $u \in H^{2}\left(M^{2 n} ; \mathbb{Z}\right)$ such that $u^{n}=k\left[M^{2 n}\right]$ and $n \nmid 4 k^{2}$.

The following result shows that the class $\mathcal{M}$ is rather wide.

Proposition 1. (1) Suppose $M^{2 n} \in \mathcal{M}$ and $N^{2 n}$ is a connected closed orientable topological $2 n$-dimensional manifold such that $b_{1}\left(N^{2 n}\right)=0$, and there is a continuous map $f: N^{2 n} \rightarrow M^{2 n}$ with $(\operatorname{deg} f, n)=1$. Then $N^{2 n}$ belongs to $\mathcal{M}$.

(2) Suppose $M^{2 n} \in \mathcal{M}$ and $N^{2 n}$ is a connected closed orientable topological $2 n$ dimensional manifold with $b_{1}\left(N^{2 n}\right)=0$. Then $M^{2 n} \# N^{2 n} \in \mathcal{M}$.

(3) $\mathbb{C} P^{n} \in \mathcal{M}$ for any $n \neq 1,2,4$.

(4) Suppose $M^{2 n} \subset \mathbb{C} P^{N}, n \neq 1,2,4$, is a nonsingular connected projective algebraic variety of dimension $n$ and degree $m$. If $n \nmid 4 m^{2}$ and $b_{1}\left(M^{2 n}\right)=0$, then $M^{2 n} \in \mathcal{M}$.

Proof. (1) Let $u \in H^{2}\left(M^{2 n} ; \mathbb{Z}\right)$ be an element such that $u^{n}=k\left[M^{2 n}\right]$ and $n \nmid 4 k^{2}$. By assumption, there is a continuous map $f: N^{2 n} \rightarrow M^{2 n}$ with $(\operatorname{deg} f, n)=1$. Let $v=f^{*} u \in H^{2}\left(N^{2 n} ; \mathbb{Z}\right)$. Then

$$
v^{n}=f^{*}\left(u^{n}\right)=f^{*}\left(k\left[M^{2 n}\right]\right)=k f^{*}\left[M^{2 n}\right]=k \operatorname{deg} f\left[N^{2 n}\right] .
$$

Since $n \nmid 4 k^{2}$ and the integer $\operatorname{deg} f$ is relatively prime with $n$, we have that $n \nmid$ $4(k \operatorname{deg} f)^{2}=4 k^{2} \operatorname{deg}^{2} f$. Thus, $N^{2 n}$ belongs to $\mathcal{M}$. This proves the first assertion.

(2) We have $b_{1}\left(M^{2 n} \# N^{2 n}\right)=b_{1}\left(M^{2 n}\right)+b_{1}\left(N^{2 n}\right)=0$. Now the desired assertion follows from (1) because there is always a natural continuous map $f: M^{2 n} \# N^{2 n} \rightarrow M^{2 n}$ of degree 1 (collapsing $N^{2 n}$ to a point). This proves the second assertion.

(3) This assertion is obvious. Taking $u \in H^{2}\left(\mathbb{C} P^{n} ; \mathbb{Z}\right)$ as a canonical generator, we have $u^{n}=1 \cdot\left[\mathbb{C} P^{n}\right]$ and $n \nmid 4=4 \cdot 1^{2}$, if $n \neq 1,2,4$.

(4) As is well known, if the degree of a nonsingular connected algebraic $n$-dimensional subvariety $i: M^{2 n} \hookrightarrow \mathbb{C} P^{N}$ is $m$, then $v^{n}=m\left[M^{2 n}\right]$, where $v=i^{*} u \in H^{2}\left(M^{2 n} ; \mathbb{Z}\right)$ and $u \in H^{2}\left(\mathbb{C} P^{N} ; \mathbb{Z}\right)$ is the canonical generator. This immediately implies that if $n \nmid 4 m^{2}$ and $b_{1}\left(M^{2 n}\right)=0$, then $M^{2 n}$ belongs to $\mathcal{M}$. The proposition is proved.

Theorem 3. If $M^{2 n}$ belongs to $\mathcal{M}$, then it does not admit a structure of $2 H$-space.

Proof. Assume the opposite: a connected closed orientable topological $2 n$-dimensional manifold $M^{2 n}$ belongs to $\mathcal{M}$ and admits a structure of $2 H$-space. By the Borsuk criterion, any topological manifold (possibly, with boundary) is an ENR-space, and any compact ENR, by a result of F. Quinn, is homotopy equivalent to a finite CW-complex. Thus, Theorem 1 applies to the $2 H$-space $M^{2 n}$. This means that there is a 2-homomorphism $\Delta: H^{*}\left(M^{2 n} ; \mathbb{Q}\right) \rightarrow H^{*}\left(M^{2 n} ; \mathbb{Q}\right) \otimes H^{*}\left(M^{2 n} ; \mathbb{Q}\right)$ satisfying the counit axiom and mapping integral elements $\alpha \in H^{*}\left(M^{2 n} ; \mathbb{Q}\right)$ to integral elements of $H^{*}\left(M^{2 n} ; \mathbb{Q}\right) \otimes H^{*}\left(M^{2 n} ; \mathbb{Q}\right)$.

Since integral homology $H_{q}\left(M^{2 n} ; \mathbb{Z}\right)$ is finitely generated for all $q \geq 0$, we have a natural isomorphism $H^{*}\left(M^{2 n} ; \mathbb{Z}\right) \otimes \mathbb{Q}=H^{*}\left(M^{2 n} ; \mathbb{Q}\right)$. Denote the cohomology algebra $H^{*}\left(M^{2 n} ; \mathbb{Q}\right)$ by $A^{*}$. 
Let $A_{\mathbb{Z}}^{*} \subset A^{*}$ denote the subring of integral elements of $A^{*}$. It is easy to see that $A_{\mathbb{Z}}^{*}=H^{*}\left(M^{2 n} ; \mathbb{Z}\right) /$ Tor. Denote by $\gamma$ the fundamental cohomology class $\left[M^{2 n}\right] \in A_{\mathbb{Z}}^{2 n}$. Since Poincaré duality holds for closed orientable topological manifolds, we have $A_{\mathbb{Z}}^{i} \cong$ $A_{\mathbb{Z}}^{2 n-i} \cong \mathbb{Z}^{b_{i}\left(M^{2 n}\right)}=\mathbb{Z}^{b_{2 n-i}\left(M^{2 n}\right)}, 0 \leq i \leq 2 n$. Moreover, multiplication in cohomology $A_{\mathbb{Z}}^{i} \otimes A_{\mathbb{Z}}^{2 n-i} \rightarrow A_{\mathbb{Z}}^{2 n}=\mathbb{Z}\langle\gamma\rangle$ defines a nondegenerate unimodular pairing $A_{\mathbb{Z}}^{i} \otimes A_{\mathbb{Z}}^{2 n-i} \rightarrow \mathbb{Z}$, $A_{\mathbb{Z}}^{2 n-i}=\operatorname{Hom}_{\mathbb{Z}}\left(A_{\mathbb{Z}}^{i}, \mathbb{Z}\right), 0 \leq i \leq 2 n$.

Since $M^{2 n} \in \mathcal{M}$, we have $\overline{A^{1}}=\{0\}$ and there is an element $u \in H^{2}\left(M^{2 n} ; \mathbb{Z}\right)$ such that $u^{n}=k\left[M^{2 n}\right]$ and $n \nmid 4 k^{2}$. Denote by the same symbol $u$ the image of $u \in H^{2}\left(M^{2 n} ; \mathbb{Z}\right)$ under the epimorphism $H^{*}\left(M^{2 n} ; \mathbb{Z}\right) \rightarrow H^{*}\left(M^{2 n} ; \mathbb{Z}\right) /$ Tor $=A_{\mathbb{Z}}^{*}$. Thus, we have an element $u \in A_{\mathbb{Z}}^{2}$ such that $u^{n}=k \gamma$ and $n \nmid 4 k^{2}$.

Consider $u^{n-1} \in A_{\mathbb{Z}}^{2 n-2}$. Suppose a homomorphism $\varphi_{u^{n-1}} \in \operatorname{Hom}_{\mathbb{Z}}\left(A_{\mathbb{Z}}^{2}, \mathbb{Z}\right)$ corresponds to $u^{n-1} \in A_{\mathbb{Z}}^{2 n-2}$ under the canonical isomorphism $A_{\mathbb{Z}}^{2 n-2} \cong \operatorname{Hom}_{\mathbb{Z}}\left(A_{\mathbb{Z}}^{2}, \mathbb{Z}\right)$. The subgroup $\operatorname{Ker} \varphi_{u^{n-1}} \subset A_{\mathbb{Z}}^{2}$ is free abelian as a subgroup of the free abelian group $A_{\mathbb{Z}}^{2}$. It is easy to see that $\operatorname{rk}\left(\operatorname{Ker} \varphi_{u^{n-1}}\right)=\operatorname{rk}\left(A_{\mathbb{Z}}^{2}\right)-1=b_{2}\left(M^{2 n}\right)-1$. Let $b_{2}\left(M^{2 n}\right)=N$.

By the structure theorem for finitely generated abelian groups, for any free finitely generated abelian group $F$ and its (free abelian) subgroup $G \subset F$ there is a basis $f_{1}, \ldots, f_{l}$ of $F$ and numbers $\mu_{1}, \ldots, \mu_{m} \in \mathbb{N}, m=\operatorname{rk}(G) \leq \operatorname{rk}(F)=l$ such that $\mu_{1} f_{1}, \ldots, \mu_{m} f_{m} \in F$ is a basis of $G$. Therefore, there is a basis $v_{1}, v_{2}, \ldots, v_{N}$ of $A_{\mathbb{Z}}^{2}$ such that $\mu_{2} v_{2}, \ldots, \mu_{N} v_{N}$ is a basis of $\operatorname{Ker} \varphi_{u^{n-1}}$ for some $\mu_{2}, \ldots, \mu_{N} \in \mathbb{N}$. But since $\varphi_{u^{n-1}}\left(\mu_{j} v_{j}\right)=\mu_{j} \varphi_{u^{n-1}}\left(v_{j}\right)=0$, $2 \leq j \leq N$, we also have $\varphi_{u^{n-1}}\left(v_{j}\right)=0,2 \leq j \leq N$, and therefore, $\mu_{2}=\ldots=\mu_{N}=1$. Thus, we have a basis $v_{1}, v_{2}, \ldots, v_{N}$ of $A_{\mathbb{Z}}^{2}$ such that $v_{2}, \ldots, v_{N}$ is a basis of $\operatorname{Ker} \varphi_{u^{n-1}}$, i.e., $u^{n-1} v_{2}=\ldots=u^{n-1} v_{N}=0$ and $u^{n-1} v_{1} \neq 0$.

Write the element $u \in A_{\mathbb{Z}}^{2}$ as a linear combination of the basis vectors $v_{1}, v_{2}, \ldots, v_{N}$ : $u=k_{1} v_{1}+k_{2} v_{2}+\ldots+k_{N} v_{N}, k_{i} \in \mathbb{Z}, 1 \leq i \leq N$. Clearly, $k_{1} \neq 0$. It is also clear that $u, v_{2}, \ldots, v_{N}$ is a basis of $A^{2}=A_{\mathbb{Z}}^{2} \otimes \mathbb{Q}$. By Theorem 1 , applied to the $2 H$ space $M^{2 n}$, there is a $\mathbb{Z}$-linear map $\Delta_{\mathbb{Z}}: A_{\mathbb{Z}}^{*} \rightarrow A_{\mathbb{Z}}^{*} \otimes A_{\mathbb{Z}}^{*}$ such that its $\mathbb{Q}$-linear extension $\Delta=\Delta_{\mathbb{Z}} \otimes \mathrm{Id}: A^{*}=A_{\mathbb{Z}}^{*} \otimes \mathbb{Q} \rightarrow A^{*} \otimes A^{*}=\left(A_{\mathbb{Z}}^{*} \otimes \mathbb{Q}\right) \otimes\left(A_{\mathbb{Z}}^{*} \otimes \mathbb{Q}\right)=\left(A_{\mathbb{Z}}^{*} \otimes A_{\mathbb{Z}}^{*}\right) \otimes \mathbb{Q}$ is a 2-homomorphism and satisfies the counit axiom.

Let $f=\frac{1}{2} \Delta$. The condition $\Phi_{3}(\Delta)(a, b, c)=0 \forall a, b, c \in A^{2 *}$, rewritten in terms of $f$, yields

$$
f(a b c)=-2 f(a) f(b) f(c)+f(a b) f(c)+f(a c) f(b)+f(b c) f(a) \quad \forall a, b, c \in A^{2 *} .
$$

It follows from the counit axiom that

$$
\begin{gathered}
f(u)=u \otimes 1+1 \otimes u, \\
f\left(u^{2}\right)=u^{2} \otimes 1+\alpha u \otimes u+\sum_{j=2}^{N} \varepsilon_{j} v_{j} \otimes u+\sum_{j=2}^{N} \delta_{j} u \otimes v_{j}+\sum_{i, j=2}^{N} \omega_{i j} v_{i} \otimes v_{j}+1 \otimes u^{2},
\end{gathered}
$$

for some constants $\alpha, \varepsilon_{*}, \delta_{*}, \omega_{* *} \in \mathbb{Q}$.

It follows easily from the theory of nongraded $k$-homomorphisms that, for any natural numbers $k \geq 1$ and $m \geq k+1$, any $\mathbb{Q}$-algebra $k$-homomorphism $g: A^{*} \rightarrow B^{*}$, and any homogeneous element $a \in A^{2 *}$, we have $g\left(a^{m}\right)=P_{m, k}\left(g(a), g\left(a^{2}\right), \ldots, g\left(a^{k}\right)\right)$, where $P_{m, k}\left(z_{1}, \ldots, z_{k}\right) \in \mathbb{Q}\left[z_{1}, \ldots, z_{k}\right]$ is a universal homogeneous polynomial of degree $m$ (the degree of $z_{s}$ equals $\left.s, 1 \leq s \leq k\right)$ whose coefficients depend only on the pair $(m, k)$. More precisely, $P_{m, k}\left(z_{1}, \ldots, z_{k}\right) \in \mathbb{Q}\left[z_{1}, \ldots, z_{k}\right]$ can be determined from the identity

$$
t_{1}^{m}+t_{2}^{m}+\ldots+t_{k}^{m}=P_{m, k}\left(t_{1}+\ldots+t_{k}, t_{1}^{2}+\ldots+t_{k}^{2}, \ldots, t_{1}^{k}+\ldots+t_{k}^{k}\right),
$$

where $t_{1}, \ldots, t_{k}$ are algebraically independent over $\mathbb{Q}$ formal variables. 
Consider now the case $k=2, m=n+1, a=u \in A^{2}$, and the universal polynomial $P_{n+1,2}\left(z_{1}, z_{2}\right)=\sum_{s_{1}+2 s_{2}=n+1} c_{s_{1} s_{2}} z_{1}^{s_{1}} z_{2}^{s_{2}}, c_{* *} \in \mathbb{Q}$. We then have

$$
\begin{gathered}
0=f\left(u^{n+1}\right)=\frac{1}{2} \Delta\left(u^{n+1}\right)=\frac{1}{2} P_{n+1,2}\left(2 f(u), 2 f\left(u^{2}\right)\right)= \\
\sum_{s_{1}+2 s_{2}=n+1} 2^{s_{1}+s_{2}-1} c_{s_{1} s_{2}} f^{s_{1}}(u) f^{s_{2}}\left(u^{2}\right) .
\end{gathered}
$$

Since $f(u) \in A^{2} \otimes A^{0} \oplus A^{0} \otimes A^{2}=\left(A^{*} \otimes A^{*}\right)^{2}$ and $f\left(u^{2}\right) \in A^{4} \otimes A^{0} \oplus A^{2} \otimes A^{2} \oplus A^{0} \otimes A^{4}=$ $\left(A^{*} \otimes A^{*}\right)^{4}$, we have, for any $s_{1}, s_{2} \geq 0, s_{1}+2 s_{2}=n+1$, that

$$
f^{s_{1}}(u) f^{s_{2}}\left(u^{2}\right) \in\left(A^{2 *} \otimes A^{2 *}\right)^{2 n+2}=A^{2 n} \otimes A^{2} \oplus A^{2 n-2} \otimes A^{4} \oplus \ldots \oplus A^{2} \otimes A^{2 n} .
$$

Let $[\cdot]_{2 n, 2}:\left(A^{2 *} \otimes A^{2 *}\right)^{2 n+2} \rightarrow A^{2 n} \otimes A^{2}$ denote the canonical projector to the direct summand $A^{2 n} \otimes A^{2} \subset\left(A^{2 *} \otimes A^{2 *}\right)^{2 n+2}$. By (3),

$$
\sum_{s_{1}+2 s_{2}=n+1} 2^{s_{1}+s_{2}-1} c_{s_{1} s_{2}}\left[f^{s_{1}}(u) f^{s_{2}}\left(u^{2}\right)\right]_{2 n, 2}=0 .
$$

We know that $A^{2 n}=\mathbb{Q}\langle\gamma\rangle=\mathbb{Q}\left\langle u^{n}\right\rangle$ and $A^{2}=\mathbb{Q}\left\langle u, v_{2}, \ldots, v_{N}\right\rangle$. Therefore, the elements $u^{n} \otimes u, u^{n} \otimes v_{2}, \ldots, u^{n} \otimes v_{N}$ form a $\mathbb{Q}$-basis of $A^{2 n} \otimes A^{2}$. Taking into account (2), we rewrite (4) as

$$
\begin{gathered}
\sum_{s_{1}+2 s_{2}=n+1} 2^{s_{1}+s_{2}-1} c_{s_{1} s_{2}}\left[f^{s_{1}}(u) f^{s_{2}}\left(u^{2}\right)\right]_{2 n, 2}=Q_{n+1}\left(\alpha, \varepsilon_{*}, \delta_{*}, \omega_{* *}\right) u^{n} \otimes u+ \\
R_{2, n+1}\left(\alpha, \varepsilon_{*}, \delta_{*}, \omega_{* *}\right) u^{n} \otimes v_{2}+\ldots+R_{N, n+1}\left(\alpha, \varepsilon_{*}, \delta_{*}, \omega_{* *}\right) u^{n} \otimes v_{N}=0 .
\end{gathered}
$$

It now follows that

$$
Q_{n+1}\left(\alpha, \varepsilon_{*}, \delta_{*}, \omega_{* *}\right)=0 .
$$

We want to show that the rational constant $Q_{n+1}\left(\alpha, \varepsilon_{*}, \delta_{*}, \omega_{* *}\right)$ depends only on $\alpha$ and does not depend on $\varepsilon_{*}, \delta_{*}$ and $\omega_{* *}$.

Indeed, $Q_{n+1}\left(\alpha, \varepsilon_{*}, \delta_{*}, \omega_{* *}\right)$ is a sum of the first coordinates of the elements $\left[f^{s_{1}}(u) f^{s_{2}}\left(u^{2}\right)\right]_{2 n, 2} \in A^{2 n} \otimes A^{2}, s_{1}+2 s_{2}=n+1$ with certain universal coefficients (in the basis $u^{n} \otimes u, u^{n} \otimes v_{2}, \ldots, u^{n} \otimes v_{N}$ of $\left.A^{2 n} \otimes A^{2}\right)$. We now examine in more detail the expression for the first coordinate of $\left[f^{s_{1}}(u) f^{s_{2}}\left(u^{2}\right)\right]_{2 n, 2}, s_{1}+2 s_{2}=n+1$. If $s_{2}=0$ and $s_{1}=n+1$, then the first coordinate of $\left[f^{n+1}(u)\right]_{2 n, 2}$ depends only on $n$.

Suppose $s_{2} \geq 1$. Distributing all products in $f^{s_{1}}(u) f^{s_{2}}\left(u^{2}\right)$, we can see that, in the resulting sum of the monomials of the form $c_{*} u^{q} v_{j_{1}} \ldots v_{j_{s}} \otimes u^{q^{\prime}} v_{j_{1}^{\prime}} \ldots v_{j_{s}^{\prime}}$, the monomials in $A^{2 n} \otimes A^{2}$ are either products of only one monomial of the form $\alpha u \otimes u, \varepsilon_{j} v_{j} \otimes u$, $2 \leq j \leq N, \delta_{j} u \otimes v_{j}, 2 \leq j \leq N, \omega_{i j} v_{i} \otimes v_{j}, 2 \leq i, j \leq N$, and the remaining $s_{1}$ monomials $u \otimes 1$ and $\left(s_{2}-1\right)$ monomials $u^{2} \otimes 1$, or products of $\left(s_{1}-1\right)$ monomials $u \otimes 1$, one monomial $1 \otimes u$, and $s_{2}$ monomials $u^{2} \otimes 1$.

The summands appearing in the second case depend only on $n$. In the first case, any summand belongs to one of the following four types:
(A) $\alpha u^{n} \otimes u$;
(B) $\varepsilon_{j} u^{n-1} v_{j} \otimes u, 2 \leq j \leq N$;
(C) $\delta_{j} u^{n} \otimes v_{j}, 2 \leq j \leq N$;
(D) $\omega_{i j} u^{n-1} v_{i} \otimes v_{j}, 2 \leq i, j \leq N$.

Since $u^{n-1} v_{2}=\ldots=u^{n-1} v_{N}=0$, the summands of types (B) and (D) are zero. Thus, we only have summands of types (A) and (C). But it is easy to see that only elements of type (A) contribute to the first coordinate of $\left[f^{s_{1}}(u) f^{s_{2}}\left(u^{2}\right)\right]_{2 n, 2}$. Such elements depend only on $\alpha$. Thus, we have shown that $Q_{n+1}\left(\alpha, \varepsilon_{*}, \delta_{*}, \omega_{* *}\right)$ depends only on $\alpha$. 
Now let us compute this constant

$$
Q_{n+1}(\alpha)=Q_{n+1}\left(\alpha, \varepsilon_{*}, \delta_{*}, \omega_{* *}\right)=Q_{n+1}(\alpha, 0,0,0) .
$$

Consider the algebra $\mathbb{Q}[\tilde{u}]$ with $\operatorname{deg} \tilde{u}=2$.

By the so-called completeness property of Frobenius $m$-homomorphisms, we have that, for any even-graded commutative $\mathbb{Q}$-algebra $B^{2 *}$ and any homogeneous elements $b_{1} \in B^{2}$, $b_{2} \in B^{4}, \ldots, b_{m} \in B^{2 m}$, there is a unique $m$-homomorphism $g_{b_{1}, b_{2}, \ldots, b_{m}}: \mathbb{Q}[\tilde{u}] \rightarrow B^{2 *}$ such that $g_{b_{1}, b_{2}, \ldots, b_{m}}\left(\tilde{u}^{k}\right)=b_{k}, 1 \leq k \leq m$ (see [5. Theorem 4.1]). Now take the algebra $A^{2 *} \otimes A^{2 *}$ as $B^{2 *}$. Then there is a unique 2-homomorphism $\widetilde{\Delta}: \mathbb{Q}[\tilde{u}] \rightarrow A^{2 *} \otimes A^{2 *}$ such that $\widetilde{\Delta}(\tilde{u})=2 u \otimes 1+1 \otimes 2 u$ and $\widetilde{\Delta}\left(\tilde{u}^{2}\right)=2 u^{2} \otimes 1+2 \alpha u \otimes u+1 \otimes 2 u^{2}$.

Consider the map $\tilde{f}=\frac{1}{2} \widetilde{\Delta}: \mathbb{Q}[\tilde{u}] \rightarrow A^{2 *} \otimes A^{2 *}$. Using the same arguments as for $f$, we have

$$
\begin{aligned}
& {\left[\tilde{f}\left(\tilde{u}^{n+1}\right)\right]_{2 n, 2}=\sum_{s_{1}+2 s_{2}=n+1} 2^{s_{1}+s_{2}-1} c_{s_{1} s_{2}}\left[\tilde{f}^{s_{1}}(\tilde{u}) \tilde{f}^{s_{2}}\left(\tilde{u}^{2}\right)\right]_{2 n, 2}=} \\
& Q_{n+1}(\alpha) u^{n} \otimes u+R_{2, n+1}(\alpha, 0,0,0) u^{n} \otimes v_{2}+\ldots+R_{N, n+1}(\alpha, 0,0,0) u^{n} \otimes v_{N} .
\end{aligned}
$$

Moreover, it is easy to see that

$$
\tilde{f}^{s_{1}}(\tilde{u}) \tilde{f}^{s_{2}}\left(\tilde{u}^{2}\right)=\sum_{k=0}^{s_{1}+2 s_{2}} d_{s_{1} s_{2} ; k}(\alpha) u^{s_{1}+2 s_{2}-k} \otimes u^{k} \quad \forall s_{1}, s_{2} \geq 0 .
$$

Thus, we have

$$
\begin{gathered}
\tilde{f}(\tilde{u})=u \otimes 1+1 \otimes u, \quad \tilde{f}\left(\tilde{u}^{2}\right)=u^{2} \otimes 1+\alpha u \otimes u+1 \otimes u^{2} ; \\
\tilde{f}\left(\tilde{u}^{m}\right)=u^{m} \otimes 1+Q_{m}(\alpha) u^{m-1} \otimes u+R_{m}(\alpha) \cdot 1 \otimes u^{2}, \quad m \geq 2,
\end{gathered}
$$

where $Q_{m}(\alpha) \in \mathbb{Q}$ and $R_{m}(\alpha) \in A^{2 *} \otimes A^{2 *}, m \geq 2$.

Also, since $2 \tilde{f}: \mathbb{Q}[\tilde{u}] \rightarrow A^{2 *} \otimes A^{2 *}$ is a 2-homomorphism, the identity $\Phi_{3}(2 \tilde{f})(a, b, c)=0$ $\forall a, b, c \in \mathbb{Q}[\tilde{u}]$ implies that

$$
\begin{gathered}
\tilde{f}(a b c)=-2 \tilde{f}(a) \tilde{f}(b) \tilde{f}(c)+\tilde{f}(a b) \tilde{f}(c)+\tilde{f}(a c) \tilde{f}(b)+\tilde{f}(b c) \tilde{f}(a) \\
\forall a, b, c \in \mathbb{Q}[\tilde{u}] .
\end{gathered}
$$

Substituting $a=b=c=\tilde{u}$ in (7), we have

$$
\tilde{f}\left(\tilde{u}^{3}\right)=u^{3} \otimes 1+3(\alpha-1) u^{2} \otimes u+R_{3}(\alpha) \cdot 1 \otimes u^{2} .
$$

Substituting $a=b=\tilde{u}, c=\tilde{u}^{m}, m \geq 2$ in (7), we have

$$
\begin{gathered}
\tilde{f}\left(\tilde{u}^{m+2}\right)=-2 \tilde{f}^{2}(\tilde{u}) \tilde{f}\left(\tilde{u}^{m}\right)+2 \tilde{f}(\tilde{u}) \tilde{f}\left(\tilde{u}^{m+1}\right)+\tilde{f}\left(\tilde{u}^{2}\right) \tilde{f}\left(\tilde{u}^{m}\right) ; \\
\tilde{f}\left(\tilde{u}^{m+2}\right)=\left(-u^{2} \otimes 1-1 \otimes u^{2}+(\alpha-4) u \otimes u\right) \times \\
\left(u^{m} \otimes 1+Q_{m}(\alpha) u^{m-1} \otimes u+R_{m}(\alpha) \cdot 1 \otimes u^{2}\right)+2(u \otimes 1+1 \otimes u) \times \\
\left(u^{m+1} \otimes 1+Q_{m+1}(\alpha) u^{m} \otimes u+R_{m+1}(\alpha) \cdot 1 \otimes u^{2}\right) .
\end{gathered}
$$

Distributing and collecting similar terms, we have a recurrence relation

$$
Q_{m+2}(\alpha)=2 Q_{m+1}(\alpha)-Q_{m}(\alpha)+\alpha-2, \quad m \geq 2 .
$$

We also know the initial conditions: $Q_{2}(\alpha)=\alpha, Q_{3}(\alpha)=3 \alpha-3$.

Together with (8), this yields $Q_{m}(\alpha)=a_{m} \alpha+b_{m}$ for some $a_{m}, b_{m} \in \mathbb{Z}, m \geq 2$. For the numbers $a_{m}$ and $b_{m}, m \geq 2$, we have, from (8), recurrence relations with constant coefficients

$$
\begin{cases}a_{m+2}=2 a_{m+1}-a_{m}+1, & m \geq 2 ; \\ b_{m+2}=2 b_{m+1}-b_{m}-2, & m \geq 2 .\end{cases}
$$


Since the initial conditions $a_{2}=1, a_{3}=3, b_{2}=0, b_{3}=-3$ are given, the above system has a unique solution, which can be found by standard methods: $a_{m}=\frac{1}{2}\left(m^{2}-m\right)$ and $b_{m}=-m^{2}+2 m \forall m \geq 2$. Thus,

$$
Q_{m}(\alpha)=\frac{1}{2} m(m-1) \alpha-m(m-2) \quad \forall m \geq 2 .
$$

As a result, we have determined the coefficient $Q_{n+1}(\alpha)=(n+1)\left(\frac{n}{2} \alpha-(n-1)\right)$. But earlier in the proof of this theorem we had $Q_{n+1}(\alpha)=Q_{n+1}\left(\alpha, \varepsilon_{*}, \delta_{*}, \omega_{* *}\right)=0$. Therefore, $\alpha=\frac{2(n-1)}{n} \in \mathbb{Q}$.

Returning to (2), we have

$$
f\left(u^{2}\right)=u^{2} \otimes 1+\alpha u \otimes u+\sum_{j=2}^{N} \varepsilon_{j} v_{j} \otimes u+\sum_{j=2}^{N} \delta_{j} u \otimes v_{j}+\sum_{i, j=2}^{N} \omega_{i j} v_{i} \otimes v_{j}+1 \otimes u^{2} .
$$

We know that $A_{\mathbb{Z}}^{2}=\mathbb{Z}\left\langle v_{1}, v_{2}, \ldots, v_{N}\right\rangle$ and $u=k_{1} v_{1}+k_{2} v_{2}+\ldots+k_{N} v_{N}, k_{i} \in \mathbb{Z}$, $1 \leq i \leq N, k_{1} \neq 0$.

Since $\Delta\left(A_{\mathbb{Z}}^{*}\right)=2 f\left(A_{\mathbb{Z}}^{*}\right) \subset A_{\mathbb{Z}}^{*} \otimes A_{\mathbb{Z}}^{*}$, we have $2 f\left(u^{2}\right) \in A_{\mathbb{Z}}^{4} \otimes A_{\mathbb{Z}}^{0} \oplus A_{\mathbb{Z}}^{2} \otimes A_{\mathbb{Z}}^{2} \oplus A_{\mathbb{Z}}^{0} \otimes A_{\mathbb{Z}}^{4}$. Consider the summand $\left[2 f\left(u^{2}\right)\right]_{2,2} \in A_{\mathbb{Z}}^{2} \otimes A_{\mathbb{Z}}^{2}$ :

$$
\begin{array}{r}
{\left[2 f\left(u^{2}\right)\right]_{2,2}=2 \alpha\left(k_{1} v_{1}+k_{2} v_{2}+\ldots+k_{N} v_{N}\right) \otimes\left(k_{1} v_{1}+k_{2} v_{2}+\ldots+k_{N} v_{N}\right)+} \\
\sum_{j=2}^{N} \varepsilon_{j} v_{j} \otimes\left(k_{1} v_{1}+k_{2} v_{2}+\ldots+k_{N} v_{N}\right)+\sum_{j=2}^{N} \delta_{j}\left(k_{1} v_{1}+k_{2} v_{2}+\ldots+k_{N} v_{N}\right) \otimes v_{j}+ \\
\quad \sum_{i, j=2}^{N} \omega_{i j} v_{i} \otimes v_{j} \in A_{\mathbb{Z}}^{2} \otimes A_{\mathbb{Z}}^{2}= \\
\mathbb{Z}\left\langle v_{1} \otimes v_{1}, v_{1} \otimes v_{j}, 2 \leq j \leq N, v_{j} \otimes v_{1}, 2 \leq j \leq N, v_{i} \otimes v_{j}, 2 \leq i, j \leq N\right\rangle .
\end{array}
$$

Since $\left[2 f\left(u^{2}\right)\right]_{2,2} \in A_{\mathbb{Z}}^{2} \otimes A_{\mathbb{Z}}^{2}$, we have $2 \alpha k_{1}^{2} \in \mathbb{Z}$. Since $\alpha=\frac{2(n-1)}{n}$ and $(n-1, n)=1$, we have $n \mid 4 k_{1}^{2}$. Moreover, $u=k_{1} v_{1}+k_{2} v_{2}+\ldots+k_{N} v_{N}$ and $u^{n}=k \gamma$.

Thus, $u^{n}=u u^{n-1}=\left(k_{1} v_{1}+k_{2} v_{2}+\ldots+k_{N} v_{N}\right) u^{n-1}=k_{1} v_{1} u^{n-1}=k_{1} q \gamma$, where $v_{1} u^{n-1}=q \gamma, q \in \mathbb{Z}$. Therefore, $k=k_{1} q$ for some $q \in \mathbb{Z}, q \neq 0$. We then have $n \mid 4 k^{2}=$ $4 k_{1}^{2} q^{2}$ because $n \mid 4 k_{1}^{2}$. But, by assumption, $n \nmid 4 k^{2}$. The obtained contradiction proves the theorem.

The class $\mathcal{M}$ introduced above does not contain 4-dimensional and 8-dimensional manifolds. At the same time, complex projective spaces $\mathbb{C} P^{n}$ of dimension $n \neq 1,2,4$ do belong to $\mathcal{M}$ and therefore do not admit a structure of $2 H$-space. The Riemann sphere $\mathbb{C} P^{1}$ has a structure of 2-valued algebraic groups, introduced by V. M. Buchstaber in 3 . It was shown in 2] that $\mathbb{C} P^{2}$ is not a $2 H$-space. The next theorem proves the same for $\mathbb{C} P^{4}$ and a number of other spaces.

Theorem 4. Let $X$ be a connected Hausdorff space homotopy equivalent to a $C W$ complex and having finitely generated integral homology groups $H_{q}(X ; \mathbb{Z})$ for all $q \geq 0$. Suppose that, for some natural number $m$, the first $m$ betti numbers of $X$ vanish, $b_{1}(X)=$ $b_{2}(X)=\ldots=b_{m}(X)=0$. Assume also that $b_{2 m}(X)=1$ and, if $m \geq 2$, then for any $k$, $1 \leq k \leq m-1$, either $b_{m+k}(X)=0$ or $b_{3 m-k}(X)=0$. If, for a generator $u \in H^{2 m}(X ; \mathbb{Q})$, $u^{n} \neq 0$ and $u^{n+1}=0$ for some $n \geq 2$, then $X$ is not a $2 H$-space.

Proof. Denote the ring $H^{*}(X ; \mathbb{Z}) /$ Tor by $A_{\mathbb{Z}}^{*}$. Because the homology $H_{q}(X ; \mathbb{Z}), q \geq 0$ is finitely generated, we have the canonical $\mathbb{Q}$-algebra isomorphism $A_{\mathbb{Z}}^{*} \otimes \mathbb{Q}:=A^{*} \cong$ $H^{*}(X ; \mathbb{Q})$. 
Assume that $X$ is a $2 H$-space. According to Theorem 1 , there is a $\mathbb{Z}$-linear map $\Delta_{\mathbb{Z}}: A_{\mathbb{Z}}^{*} \rightarrow A_{\mathbb{Z}}^{*} \otimes A_{\mathbb{Z}}^{*}$ such that the $\mathbb{Q}$-linear extension $\Delta=\Delta_{\mathbb{Z}} \otimes \mathrm{Id}: A^{*} \rightarrow A^{*} \otimes A^{*}$ is a 2-homomorphism and satisfies the counit axiom. It is easy to see that for the element $u \in A^{2 m}$ in the statement of the theorem we can take a generator of $A_{\mathbb{Z}}^{2 m} \subset A^{2 m}$, $A_{\mathbb{Z}}^{2 m}=\mathbb{Z}\langle u\rangle$.

Let $f=\frac{1}{2} \Delta$. By the counit axiom and the assumptions on $A^{*}$,

$$
f(u)=u \otimes 1+1 \otimes u, \quad f\left(u^{2}\right)=u^{2} \otimes 1+1 \otimes u^{2}+\alpha u \otimes u,
$$

where $\alpha \in \frac{\mathbb{Z}}{2}$ by the integrality of $\Delta$.

Using the same arguments as in the proof of Theorem 3, we have the implication $u^{n+1}=0 \Rightarrow f\left(u^{n+1}\right)=0 \Rightarrow \alpha=\frac{2(n-1)}{n}$. Since $\alpha \in \frac{\mathbb{Z}}{2}$, it follows that $n=2$ or $n=4$. Thus, for $n \neq 2,4$ and $n \geq 2$, the theorem is already proved.

Suppose $n=2$ or 4 . Formula (1) from the proof of Theorem 3, which applies to our case, yields a recurrence relation

$$
f\left(u^{m+2}\right)=-2 f^{2}(u) f\left(u^{m}\right)+2 f(u) f\left(u^{m+1}\right)+f\left(u^{2}\right) f\left(u^{m}\right) \quad \forall m \geq 1 .
$$

The initial conditions $\left(f(u)=u \otimes 1+1 \otimes u, f\left(u^{2}\right)=u^{2} \otimes 1+1 \otimes u^{2}+\alpha u \otimes u\right)$ allow us to compute $f\left(u^{m}\right)$ for all $m \geq 3$ whenever $\alpha$ is known.

Let $n=2$. Then $\alpha=\frac{2(n-1)}{n}=1$. Direct calculations show that $0=f\left(u^{4}\right)=$ $-5 u^{2} \otimes u^{2} \neq 0$. The obtained contradiction proves the theorem in the case $n=2$.

Let $n=4$. Then $\alpha=\frac{2(n-1)}{n}=\frac{3}{2}$. Calculations show that $0=f\left(u^{5}\right)=$ $-\frac{15}{4}\left(u^{3} \otimes u^{2}+u^{2} \otimes u^{3}\right) \neq 0$. The obtained contradiction proves the theorem in the remaining case $n=4$. The theorem is proved.

\section{$\S 5$. REtracts OF $n H$-SPACES}

The next lemma shows that, for $\mathrm{CW}$-complexes $X$, the homotopy unit axiom for $n$-valued multiplications $\mu: X \times X \rightarrow \operatorname{Sym}^{n} X$ is equivalent to the strong unit axiom.

Lemma 2. Let $X$ be a connected $C W$-complex, $x_{0}$ and $x_{1} \in X$ two arbitrary points of $X$, and $\mu_{0}:\left(X, x_{0}\right) \times\left(X, x_{0}\right) \rightarrow\left(\operatorname{Sym}^{n} X,\left[n x_{0}\right]\right)$ an $n$-valued multiplication for which $x_{0}$ is a homotopy unit. Then there is an n-valued multiplication $\mu_{1}:\left(X, x_{1}\right) \times\left(X, x_{1}\right) \rightarrow$ $\left(\operatorname{Sym}^{n} X,\left[n x_{1}\right]\right)$, freely homotopic to $\mu_{0}$ and such that the strong unit axiom $\mu_{1}\left(x, x_{1}\right)=$ $\mu_{1}\left(x_{1}, x\right)=[n x] \forall x \in X$ holds.

Proof. The term homotopy will refer to the homotopy of pointed spaces, as opposed to free homotopy (of non-pointed spaces). It is known that any point of an arbitrary CW-complex can be made into a vertex by subdividing a finite number of cells.

Therefore, we may assume that $x_{0}$ and $x_{1} \in X$ are vertices of the complex $X$. Since $X$ is connected, the vertices $x_{0}$ and $x_{1}$ can be connected by a continuous path $h:\left\{x_{0}\right\} \times I \rightarrow$ $X, h\left(x_{0}, 0\right)=x_{0}, h\left(x_{0}, 1\right)=x_{1}$. The pair $\left(X,\left\{x_{0}\right\}\right)$ is called a Borsuk pair. Therefore, the free homotopy $h:\left\{x_{0}\right\} \times I \rightarrow X$ extends to a free homotopy $H: X \times I \rightarrow X$ such that $H_{0}=\operatorname{Id}_{X}$ and $H_{1}\left(x_{0}\right)=x_{1}$. Denote the map $H_{1}:\left(X, x_{0}\right) \rightarrow\left(X, x_{1}\right)$ by $f$.

The map $f:\left(X, x_{0}\right) \rightarrow\left(X, x_{1}\right)$ is freely homotopic to $\operatorname{Id}_{X}$ and therefore is a homotopy equivalence. Since $X$ is a CW-complex, $f:\left(X, x_{0}\right) \rightarrow\left(X, x_{1}\right)$ is a pointed homotopy equivalence. Let $g:\left(X, x_{1}\right) \rightarrow\left(X, x_{0}\right)$ be the homotopy inverse of $f$.

Let $[F]$ denote the free homotopy class of an arbitrary map $F:\left(Y, y_{0}\right) \rightarrow\left(Z, z_{0}\right)$, and $[F]^{\bullet}$ - the pointed homotopy class of $F$. By definition, $[g]^{\bullet}=\left([f]^{\bullet}\right)^{-1}$. This implies that $[g]=[f]^{-1}=\operatorname{Id}_{X}^{-1}=\operatorname{Id}_{X}$. 
Define a continuous map $\widetilde{\mu}_{1}:\left(X, x_{1}\right) \times\left(X, x_{1}\right) \rightarrow\left(\operatorname{Sym}^{n} X,\left[n x_{1}\right]\right)$ using the following commutative diagram:

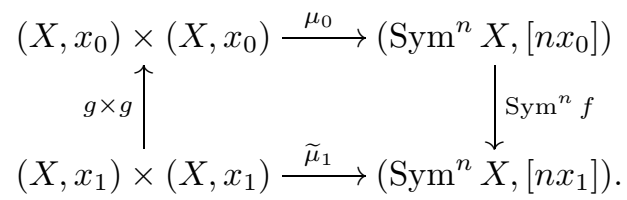

Since $\operatorname{Sym}^{n}$ is a homotopy functor, we have $\left[\operatorname{Sym}^{n} f\right]=\left[\operatorname{Sym}^{n} \operatorname{Id}_{X}\right]=\operatorname{Id}_{\operatorname{Sym}^{n} X}$. Therefore,

$$
\begin{aligned}
& {\left[\widetilde{\mu}_{1}\right]=\left[\operatorname{Sym}^{n} f \circ \mu_{0} \circ(g \times g)\right]=\left[\operatorname{Sym}^{n} f\right] \circ\left[\mu_{0}\right] \circ([g] \times[g])=} \\
& \operatorname{Id}_{\operatorname{Sym}^{n} X} \circ\left[\mu_{0}\right] \circ \operatorname{Id}_{X^{2}}=\left[\mu_{0}\right] .
\end{aligned}
$$

Define a map $\nu_{0}:\left(X \times\left\{x_{0}\right\}\right) \cup\left(\left\{x_{0}\right\} \times X\right) \rightarrow\left(\operatorname{Sym}^{n} X,\left[n x_{0}\right]\right)$ by setting $\nu_{0}\left(x, x_{0}\right)=$ $\nu_{0}\left(x_{0}, x\right)=[n x] \forall x \in X$. By assumption, $x_{0} \in X$ is a homotopy unit for the $n$-valued multiplication $\mu_{0}:\left(X, x_{0}\right) \times\left(X, x_{0}\right) \rightarrow\left(\operatorname{Sym}^{n} X,\left[n x_{0}\right]\right)$. Since the homotopy here is meant to fix $x_{0}$, this condition is equivalent to the equality $\left[\left.\mu_{0}\right|_{\left(X \times\left\{x_{0}\right\}\right) \cup\left(\left\{x_{0}\right\} \times X\right)}\right]^{\bullet}=$ $\left[\nu_{0}\right]^{\bullet}$. Denote $\left(X \times\left\{x_{i}\right\}\right) \cup\left(\left\{x_{i}\right\} \times X\right)$ by $X \vee_{x_{i}} X, i=0,1$.

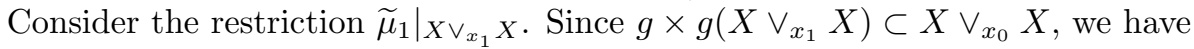

$$
\begin{aligned}
{\left[\left.\widetilde{\mu}_{1}\right|_{X \vee_{x_{1} X} X}\right]^{\bullet}=} & {\left[\operatorname{Sym}^{n} f\right]^{\bullet} \circ\left[\left.\mu_{0}\right|_{X \vee_{x_{0} X}}\right]^{\bullet} \circ\left[g \times\left. g\right|_{X \vee_{x_{1} X}}\right]^{\bullet}=} \\
& {\left[\operatorname{Sym}^{n} f\right]^{\bullet} \circ\left[\nu_{0}\right]^{\bullet} \circ\left[g \times\left. g\right|_{X \vee_{x_{1}} X}\right]^{\bullet}=\left[\operatorname{Sym}^{n} f \circ \nu_{0} \circ\left(g \times\left. g\right|_{X \vee_{x_{1}} X}\right)\right]^{\bullet} . }
\end{aligned}
$$

It is easy to check that

$$
\begin{aligned}
\left(\operatorname{Sym}^{n} f \circ \nu_{0} \circ\left(g \times\left. g\right|_{X \vee_{x_{1}} X}\right)\right)\left(x, x_{1}\right)=\left(\operatorname{Sym}^{n} f \circ \nu_{0} \circ\left(g \times\left. g\right|_{X \vee_{x_{1}} X}\right)\right)\left(x_{1}, x\right) & = \\
& {[n(f \circ g)(x)] \in \operatorname{Sym}^{n} X \quad \forall x \in X . }
\end{aligned}
$$

Since $[f \circ g]^{\bullet}=\operatorname{Id}_{\left(X, x_{1}\right)}$, the last two equalities imply that $\left[\left.\widetilde{\mu}_{1}\right|_{X \vee_{x_{1}} X}\right]^{\bullet}=\left[\nu_{1}\right]^{\bullet}$, where the map $\nu_{1}: X \vee_{x_{1}} X \rightarrow\left(\operatorname{Sym}^{n} X,\left[n x_{1}\right]\right)$ is given by $\nu_{1}\left(x, x_{1}\right)=\nu_{1}\left(x_{1}, x\right)=[n x]$ $\forall x \in X$. Thus, we have proved that $x_{1} \in X$ is a homotopy unit for the multiplication $\widetilde{\mu}_{1}:\left(X, x_{1}\right) \times\left(X, x_{1}\right) \rightarrow\left(\operatorname{Sym}^{n} X,\left[n x_{1}\right]\right)$.

The pair $\left(X \times X, X \vee_{x_{1}} X\right)$ is the product of the closed Borsuk pair $\left(X,\left\{x_{1}\right\}\right)$ with itself, and is therefore a closed Borsuk pair (see [9, p. 48]). Hence, the homotopy connecting $\left.\widetilde{\mu}_{1}\right|_{X \vee_{x_{1}} X}$ with $\nu_{1}$ can be extended from $X \vee_{x_{1}} X$ to the entire space $X \times X$. Restricting this homotopy to $t=1$, we have the desired $n$-valued multiplication $\mu_{1}:\left(X, x_{1}\right) \times$ $\left(X, x_{1}\right) \rightarrow\left(\operatorname{Sym}^{n} X,\left[n x_{1}\right]\right)$ satisfying the strong unit axiom $\mu_{1}\left(x, x_{1}\right)=\mu_{1}\left(x_{1}, x\right)=$ $[n x] \forall x \in X$. Moreover, the map $\mu_{1}$ is homotopic to $\widetilde{\mu}_{1}$ and, therefore, is freely homotopic to $\mu_{0}$. The lemma is proved.

Let us show that, for $\mathrm{CW}$-complexes, the property of being an $n H$-space is invariant under retraction.

Proposition 2. Let $X$ be a connected $C W$-complex with an $n H$-space structure for some $n \geq 1$. Then any retract $Y \subset X$ also has an $n H$-space structure.

Proof. Consider an arbitrary point $y_{0} \in Y$. By Lemma 2 , there is an $n$-valued multiplication $\mu_{X}: X \times X \rightarrow \operatorname{Sym}^{n} X$ with (a strong) unit $y_{0} \in X, \mu_{X}\left(y_{0}, x\right)=\mu_{X}\left(x, y_{0}\right)=[n x]$ $\forall x \in X$. Let $r: X \rightarrow Y$ be a retraction, $r(y)=y \forall y \in Y \subset X$. Define an $n$-valued multiplication $\mu_{Y}: Y \times Y \rightarrow \operatorname{Sym}^{n} Y$ by the formula

$$
\mu_{Y}\left(y_{1}, y_{2}\right)=\operatorname{Sym}^{n} r \circ \mu_{X}\left(y_{1}, y_{2}\right) \quad \forall y_{1}, y_{2} \in Y \text {. }
$$

The continuity of $\mu_{Y}$ is obvious. Moreover,

$$
\mu_{Y}\left(y_{0}, y\right)=\operatorname{Sym}^{n} r \circ \mu_{X}\left(y_{0}, y\right)=\operatorname{Sym}^{n} r([n y])=[n y] \in \operatorname{Sym}^{n} Y \quad \forall y \in Y .
$$


Similarly, $\mu_{Y}\left(y, y_{0}\right)=[n y] \in \operatorname{Sym}^{n} Y \forall y \in Y$. Thus, we have defined an $n$-valued multiplication $\mu_{Y}: Y \times Y \rightarrow \operatorname{Sym}^{n} Y$ with identity $y_{0} \in Y$. The proposition is proved.

Proposition 2 implies the following

Proposition 3. Let $X$ be a connected $C W$-complex having a retract $Y \subset X, r: X \rightarrow Y$ such that either $Y$ belongs to the class $\mathcal{M}$ introduced above or $Y$ satisfies the conditions of Theorem 4. Then $X$ does not admit a 2-valued multiplication with identity.

\section{ACKNOWLEDGMENTS}

The author is deeply grateful to the Corresponding Member of the Russian Academy of Sciences Professor V. M. Buchstaber for posing this problem and for his interest in this work. The author is also grateful to the Lead Researcher A. A. Gaifullin and to the Senior Researcher S. A. Melikhov for valuable discussions.

\section{REFERENCES}

[1] V. M. Buchstaber, n-valued groups: theory and applications, Mosc. Math. J. 6 (2006), no. 1, 57-84. MR2265947 (2007i:20102)

[2] V. M. Buchstaber and E. G. Rees, Multivalued groups, their representations and Hopf algebras, Transform. Groups 2 (1997), no. 4, 325-349. MR1486035 (99b:20071)

[3] V. M. Buchstaber, Functional equations that are associated with addition theorems for elliptic functions, and two-valued algebraic groups, Uspekhi Mat. Nauk 45 (1990), no. 3 (273), 185-186; English transl., Russian Math. Surveys 45 (1990), no. 3, 213-215. MR1071939 (91j:39002)

[4] D. V. Gugnin, Topological applications of graded Frobenius n-homomorphisms, Tr. Mosk. Mat. Obs. 72 (2011), no. 1, 127-188; English transl., Trans. Moscow Math. Soc. 72 (2011), no. 1, 97-142.

[5] D. V. Gugnin, Polynomially dependent homomorphisms and Frobenius n-homomorphisms, Tr. Mat. Inst. Steklova 266 (2009), Geometriya, Topologiya i Matematicheskaya Fizika. II, 64-96; English transl., Proc. Steklov Inst. Math. 266 (2009), no. 1, 59-90. MR2603261 (2011e:16077)

[6] A. Grothendieck, Sur quelques points d'algèbre homologique, Tôhoku Math. J. (2) 9 (1957), 119-221. MR0102537 (21:1328)

[7] I. G. Macdonald, The Poincaré polynomial of a symmetric product, Proc. Cambridge Philos. Soc. 58 (1962), 563-568. MR0143204 (26:764)

[8] I. G. Macdonald, Symmetric products of an algebraic curve, Topology 1 (1962), 319-343. MR0151460 $(27: 1445)$

[9] M. M. Postnikov, Lectures in algebraic topology. Elements of homotopy theory, Nauka, Moscow, 1984. (Russian) MR:776974 (87j:55002)

Chair of Higher Geometry and Topology, Department of Mechanics and Mathematics, Moscow State University, Moscow, Russia

E-mail address: dmitry-gugnin@yandex.ru 